\title{
Voltammetry of Adsorbed Species: Non- ideal Interactions Leading to Phase Transitions
}

\author{
Minjun Yang1, Richard G. Compton ${ }^{1 *}\left(\Xi_{\circledast}\right)$ \\ ${ }^{1}$ Department of Chemistry, Physical and Theoretical Chemistry Laboratory, University of Oxford, South Parks Road, Oxford \\ OX1 3QZ, United Kingdom \\ * Corresponding author email: Richard.Compton@chem.ox.ac.uk
}

Abstract: Phase transition behaviours are not uncommon in voltammetry owning to strong interactions on the surface. By coupling interfacial electrode kinetics with Frumkin adsorption isotherm, the underlining physicochemical processes occurring in voltammetry induced phase transition are studied via simulation herein. We report phase-transition-like behaviour is only observable in voltammetry under reversible electrode kinetic, which otherwise, would lead to a 'disconnection' from the Frumkin adsorption isotherm. In case when phase transition occurs in the timescale of voltammetry, the simulated interfacial conditions reveal intrusions into the 'forbidden' part predicted by the Frumkin adsorption isotherm, which is otherwise inaccessible to the prediction on the assumption of mass transport equilibrium, even if significant excess in bulk concentration is used.

\section{Keywords}

Phase Transition; Voltammetry; Frumkin Adsorption Isotherm; Electrochemical Interfaces;

Electrochemistry; 


\section{Introduction}

The understanding of electrochemical interfaces significantly advanced during the twentieth century partly because of the extensive electrocapillary measurements made on a mercury-water interface in the presence of organic molecules. ${ }^{1-3}$ The most notable contributor in this area was Alexander $\mathrm{N}$. Frumkin, a Soviet electrochemist who contributed remarkably in this field and is widely known as the 'father of modern electrochemistry' for his pioneering work. ${ }^{4}$ His most recognised works include but are not restricted to, identifying the potential of zero charge, providing an answer to the origin of electromotive forces and settling a long dispute between Volta and Nernst. ${ }^{5}$ Furthermore, Frumkin development an adsorption isotherm, bearing his name, to explain the experimental data obtained by him and his co-workers' on the adsorption of various organic molecules on mercury by accounting for non-ideality by including a surface interaction parameter. ${ }^{6}$ Since then, a wide range of alternative adsorption isotherms have been developed with varying degrees of complexity ${ }^{7-9}$. Nevertheless, the adsorption isotherm developed by Frumkin remains one of the best in giving agreement with a diversity of large sets of experimental data. ${ }^{7,10}$

Accounting for surface interactions is vital for voltammetry involving adsorbed species as it plays a crucial role in the influencing both the thermodynamics and the rate of interfacial reactions. For a simple one-electron transfer redox couple, either both, one or zero of the reactant and product can be adsorbed on the electrode surface; the former case was studied extensively in the seminal work by Laviron et al. where the inclusion of the Frumkin adsorption isotherm accounts for the lateral surface interactions for a strongly adsorbed redox couple. ${ }^{11-13}$ More recent advances in the modelling of this diffusionless system include an approximate calculation algorithm allowing this to be studied in Excel, albeit having nine adjustable parameters, without the use of programming languages such as $C .{ }^{14}$

Redox couples involving one strongly adsorbed species (Equation 1) are common in a vast number of electrochemical processes covering a wide range of redox reactions..$^{15-17}$ In particular under-potential 
deposition (UPD) of metal atoms on a foreign surface leading to a monolayer of adatom is relevant across many scientific and technological disciplines involving materials, surface and catalysis scientific. Over recent decades, advances in the preparation of pristine single-crystal surfaces allow surface interactions to be studied on individual facets of the same electrode material. This includes, but is not limited to, under-potential deposition of the following adatom layers; $\mathrm{H}^{18-20} \mathrm{Cu}^{21-24}$ and $\mathrm{Ag}^{25-27}$ on $\mathrm{Pt}$ electrodes; $\mathrm{Cu}^{28-30} \mathrm{Ag}{ }^{31-33} \mathrm{~Pb}^{34-36}$ and $\mathrm{Hg}^{37-39}$ on Au electrodes; $\mathrm{Tl}^{40-41}$ and $\mathrm{Pb}^{42-44}$ on $\mathrm{Ag}$ electrodes. Notice that, the formation of adatom in UPD reactions, or generally termed electro-sorption reactions in this article (Equation 1), is a concerted step accompanying charge-transfer across the interface, different to that of a stepwise adsorption kinetics of the reactant followed by charge-transfer. Beyond the niche substrate of single-crystal surfaces, which are typically somewhat exclusively studied, UPD can occur with liquid mercury electrodes leading to the formation of $\mathrm{HgS}$ monolayers. ${ }^{45-47}$ It is not uncommon that UPD results in abnormally narrow and sharp voltammetry peaks. ${ }^{42-43,45}$ To understand this, simulations accounting for surface interactions are necessary. A common approach is an interpretation of the peak width in terms of the magnitude of the surface interactions. In the literature, this problem is simplified by assuming a diffusionless system, i.e. $c_{\mathrm{A}, 0}=c_{\mathrm{A}, \text { bulk }}$ where $c$ is the solution phase concentration and the subscripts denote either the surface or bulk values, at all times, justified experimentally by the use of a high concentration of the reactant $(0.1 \mathrm{M})$ and extreme slow voltage scan rates $\left(0.1 \mathrm{mVs}^{-1}\right) \cdot{ }^{43}, 45,48-49$ Of those studies accounting for mass transport, Baranski et al. modelled the surface process as a transfer of the adsorbate from the outer- to the inner-Helmholtz plane coupled with Frumkin adsorption isotherm..$^{50}$ whilst Fawcet et al. focused on the desorption process..$^{51}$

This article simulates the voltammetry of an electro-sorption reaction involving one species strongly adsorbed on the electrode surface. We take a bottom-up approach in developing a mathematical model coupling the Frumkin adsorption isotherm with Butler-Volmer kinetics to describe the surface electrode kinetics. The underlining physicochemical processes and effects of surface interactions are studied and, in particularly, consider the strong interactions leading to phase transition behaviours in 
voltammetry. Moreover, this article demonstrates that mass transport in the solution phase is, in fact, crucial in the interpretation of experimentally obtained voltammograms close to phase transitional surface interactions - even if a significant excess in solution concentrations and slow scan rates are deployed. 


\section{Theoretical Model}

In the following we consider a one-step electro-deposition reaction

$$
A(a q)+e^{-}+M(\text { site }) \rightleftharpoons B(\text { ads }) \quad(\text { Eq. 1) }
$$

where a solution phase species $\mathrm{A}(a q)$ gains an electron to form species $\mathrm{B}(a d s)$ adsorbed onto an unoccupied site on a macro-electrode. In the sections below, we first discuss, generally, adsorption isotherms which allow non-ideal surface interactions to be accounted for. Next, the electrode kinetics describing the heterogeneous rate constant as a function of the applied potential is developed for the case of Equation 1. This requires a bespoke formal potential $E_{f}^{0}$ to be defined, complementary to that adopted by IUPAC. Finally, a full mathematical model, including diffusional effects, for the simulation of the reaction in Equation 1 is outlined together with the definitions of the dimensionless parameters used to report results.

\subsection{Theory}

\section{Adsorption isotherms}

First, we consider a general adsorption process where a species A, either in the gas phase or aqueous phase, adsorbs onto a surface with unoccupied equivalent sites $\mathrm{M}$ (site) becoming surface-bound $\mathrm{A}($ ads $)$

$$
\mathrm{A}+\mathrm{M}(\text { site }) \stackrel{k_{a d s}}{\stackrel{2}{\rightleftharpoons}} \mathrm{A}(\text { ads })
$$

where $k_{a d s}$ is the adsorption rate constant $\left(\mathrm{m}^{3} \mathrm{~mol}^{-1} \mathrm{~s}^{-1}\right), k_{\text {des }}$ is the desorption rate constant $\left(\mathrm{s}^{-1}\right)$. In an ideal scenario, the adsorbates on the surface do not interact, all sites are identical and can only one adsorbate occupy one site. The rate of adsorption is then directly proportional to an adsorption rate constant $k_{a d s}$, the interfacial concentration of $c_{\mathrm{A}, 0}$ and the number of unoccupied sites $\left(\Gamma_{\max }-\Gamma_{\mathrm{A}}\right)$. Similarly, the rate of desorption will be directly proportional to the desorption rate constant $k_{d e s}$ and the number of surface-bound $\mathrm{A}\left(\Gamma_{\mathrm{A}}\right)$, giving rise to a general net rate law 


$$
\text { rate }=\frac{d \Gamma_{\mathrm{A}}}{d t}=k_{a d s} c_{\mathrm{A}, 0}\left(\Gamma_{\max }-\Gamma_{\mathrm{A}}\right)-k_{d e s} \Gamma_{\mathrm{A}} \quad(\text { Eq. 3) }
$$

where $t$ is time $(\mathrm{s}), c_{\mathrm{A}, 0}$ is the interfacial concentration of $\mathrm{A}\left(\mathrm{mol} \mathrm{m}^{-3}\right), \Gamma_{\max }$ is the total number of sites on the surface $\left(\mathrm{mol} \mathrm{m}^{-2}\right)$ and $\Gamma_{\mathrm{A}}$ is the coverage of the sites occupied by $\mathrm{A}\left(\mathrm{mol} \mathrm{m}^{-2}\right)$. When equilibrium between the surface and the bulk solution is established, the net rate of adsorption and desorption is zero, returning the Langmuir ideal adsorption isotherm ${ }^{52}$

$$
\frac{k_{a d s}}{k_{d e s}} c_{\mathrm{A}, 0}=\frac{\theta}{1-\theta} \quad \text { (Eq. 4) }
$$

where $\theta$ is the fractional surface coverage $\left(\frac{\Gamma_{\mathrm{A}}}{\Gamma_{\max }}\right)$ and $c_{\mathrm{A}, 0}$ equals $c_{\mathrm{A}, \text { bulk }}$ at system equilibrium. However, most aqueous soluble neutral organic compounds such as aliphatic oxygen compounds, amines and aromatics adsorb strongly onto electrodes, particularly the much-studied mercury electrode. ${ }^{53-54}$ The surface interactions arising, for example, from dipole-dipole interactions between the neighbouring adsorbates ${ }^{55-57}$, and, in the case of aromatic compounds, $\pi$-electrons interaction with mercury electrode, ${ }^{58-60}$ are far from negligible. In most cases, such as those classically observed in electrocapillary experiments on mercury, the net interaction on the surface is not zero. To account for these non-ideal surface interactions, Frumkin ${ }^{4}$ developed the adsorption isotherm bearing his name in $1926^{61}$

$$
\frac{k_{a d s}}{k_{d e s}} c_{\mathrm{A}, 0}=\frac{\theta}{1-\theta} \exp (g \theta)
$$

where $g$ is the "Frumkin adsorbate-adsorbate interaction parameter" and again, $c_{\mathrm{A}, 0}$ equals $c_{\mathrm{A}, b u l k}$ at system equilibrium. Since the isotherm describes the surface coverage versus bulk concentration at equilibrium, the position of this equilibrium is necessary controlled by the Gibb's energy of adsorption. One of the approximations leading to the derivation of Frumkin isotherm is that the adsorbatesubstrate interactions contributing to the Gibb's free energy of adsorption $\left(\Delta G_{\theta}^{\circ}\right)$ is independent to the coverage of adsorbate, but that arising from adsorbate-adsorbate interactions, is however, dependent and assumed to vary linearly with coverage $\left(\Delta G_{\theta}^{\circ}=\Delta G_{\theta=0}^{\circ}-g \theta\right) \cdot{ }^{62-63}$ In this article, the 
term non-ideal surface interaction refers to a non-zero adsorbate-adsorbate interaction parameter $g$, which could either be positive or negative. A positive $g$ indicates repulsion between the surfacebound species and a negative $g$ attractive forces. Note, the Langmuir ideal adsorption isotherm is a special case of Frumkin's adsorption isotherm when $g$ equals zero. It can be seen from the adsorption isotherm that when $g$ is small, and the contribution from the exponential term is low, the fractional surface coverage $\theta$ at equilibrium will increase with $c_{\mathrm{A}, \text { bulk }}$; however, when the surface interaction is strongly attractive $(g \leq-4)$, the plot of $\theta$ vs $c_{\mathrm{A}, b u l k}$ becomes less intuitive with indications of phase transition like behaviour, of which more below. ${ }^{1}$ The adsorption-desorption rate law accounting for the non-ideal interactions underpinning the Frumkin isotherm is ${ }^{50-51}$

$$
\text { rate }=\frac{d \Gamma_{\mathrm{A}}}{d t}=k_{a d s} c_{\mathrm{A}, 0}\left(\Gamma_{\max }-\Gamma_{\mathrm{A}}\right) \exp \left(-g^{\ddagger} \theta\right)-k_{\text {des }} \Gamma_{\mathrm{A}} \exp \left(\left(g-g^{\ddagger}\right) \theta\right) \quad \text { (Eq. 6) }
$$

where the additional term $g^{\ddagger}$ is introduced to account for the lateral interactions between the activated complex (transition state) and adsorbed molecules.

\section{Electrode kinetics}

For surface redox processes, the electrode kinetics can be successfully accounted for by the asymmetric Marcus-Hush theory involving a 're-organisation energy' and an asymmetry factor ${ }^{64-66}$ For small over-potentials, however, where the Tafel slope is typically linear the rates can be described by the semi-empirical Butler-Volmer kinetics. ${ }^{67-69}$ For a simple one-electron process $\mathrm{A}(a q) \pm e^{-} \rightleftharpoons$ $\mathrm{B}(a q)$ this gives simple expressions for the electron transfer rate constants $k_{o x}$ for oxidation and $k_{\text {red }}$ for reduction. These vary exponentially with the applied potential $E$ and depend on a transfer coefficient $\alpha$

$$
k_{r e d} \propto e^{-\frac{\alpha F}{R T}(E)} \text { and } k_{o x} \propto e^{\frac{(1-\alpha) F}{R T}(E)}
$$

where $T$ is the temperature (K), $R$ is the gas constant $\left(8.314 \mathrm{~J} \mathrm{~mol}^{-1} \mathrm{~K}^{-1}\right)$ and $F$ is the Faraday constant $\left(96485.3 \mathrm{C} \mathrm{mol}^{-1}\right)$. For a fully aqueous phase redox couple with a formal potential $E_{f}^{0}$ as defined by the 
IUPAC (Section 2.2), the Butler-Volmer kinetics is more conveniently and thus commonly expressed relative to the standard formal potential $\left(E-E_{f}^{0}\right)$ giving rise to

$$
k_{r e d}=k_{0} e^{-\frac{\alpha F}{R T}\left(E-E_{f}^{0}\right)} \text { and } k_{o x}=k_{0} e^{\frac{(1-\alpha) F}{R T}\left(E-E_{f}^{0}\right)}
$$

where $k_{0}$ is the standard electrochemical rate constant. An adapted form of Butler-Volmer kinetics is used here for ease of computation and because it allows exploration of non-ideal surface interactions coupled with mass transport.

Specifically, Butler-Volmer kinetics here is coupled into the adsorption-desorption rate law accounting for non-ideal surface interactions leading to the following expression for the adsorption flux of $A$ at the electrode interface ${ }^{50-51,70}$

$$
D_{A}\left(\frac{\partial c_{A}}{\partial x}\right)_{x=0}=k_{r e d}^{\prime} c_{A, 0}\left(\Gamma_{\max }-\Gamma_{B}\right) \exp \left(-g^{\ddagger} \theta_{B}\right)-k_{o x}^{\prime} \Gamma_{B} \exp \left(\left(g-g^{\ddagger}\right) \theta_{B}\right) \quad \text { (Eq. 9) }
$$

where $D_{\mathrm{A}}$ is the diffusion coefficient of species $\mathrm{A}, k_{r e d}^{\prime}\left(\mathrm{m}^{3} \mathrm{~mol}^{-1} \mathrm{~s}^{-1}\right)$ and $k_{o x}^{\prime}\left(\mathrm{s}^{-1}\right)$ are the electrosorption rate constants for the electro-adsorption and electro-desorption reaction, respectively. Herein, an apostrophe is used to differentiate electro-sorption rate constants from electron transfer rate constants. The former, analogous to Butler-Volmer kinetics, are exponentially dependent on the applied potential $E$

$$
\begin{aligned}
& k_{r e d}^{\prime}=k_{r e d}^{\prime \prime} e^{-\frac{\alpha F}{R T}(E)} \\
& k_{o x}^{\prime}=k_{o x}^{\prime \prime} e^{\frac{(1-\alpha) F}{R T}(E)}
\end{aligned}
$$

where $k_{r e d / o x}^{\prime \prime}$ is the pre-exponential constants. It is, however, more insightful to express $k_{r e d}^{\prime}$ and $k_{o x}^{\prime}$ in terms of an applied potential relative to a suitably defined formal potential.

\subsection{Definition of the Formal Potential $E_{f}^{0}$}

The definition of formal potential as adopted by IUPAC is as follows "[The] Conditional (formal) potential, $E_{c}^{0^{\prime}}$, is related to the potential of a cell reaction, $E_{c e l l}$, by an equation analogous to that 
relating $E_{\text {cell }}$ to the standard potential $E_{0}$, with the activity, $a_{i}$, replaced by any composition variable, $c_{i}$ (to be indicated by a subscript). For example: $E_{c e l l}=E_{c}^{0^{\prime}}-\frac{R T}{n F} \sum_{i} v_{i} l n c_{i} .{ }^{171}$ This expression for the formal potential $\left(E_{c}^{0^{\prime}}\right.$ or $\left.E_{f}^{0}\right)$ is not suitable for the one step electro-sorption mechanism.

Here, we find an expression for the applied potential $E$, in terms of $c_{\mathrm{A}, b u l k}, \Gamma_{\mathrm{B}}$ and a bespoke definition of formal potential $E_{f}^{0}$. When the system is in equilibrium, the adsorption flux of $A$ at the electrode interface equals zero

$$
0=k_{r e d}^{\prime} c_{\mathrm{A}, 0}\left(\Gamma_{\max }-\Gamma_{\mathrm{B}}\right) \exp \left(-g^{\ddagger} \theta_{\mathrm{B}}\right)-k_{o x}^{\prime} \Gamma_{\mathrm{B}} \exp \left(\left(g-g^{\ddagger}\right) \theta_{\mathrm{B}}\right)
$$

and the interfacial concentration $c_{\mathrm{A}, 0}$ equals to the bulk concentration $c_{\mathrm{A}, b u l k}$, so substituting Equations 10 and 11 into Equation 12 and rearranging gives

$k_{\text {red }}^{\prime \prime} e^{-\frac{\alpha F}{R T} E} c_{\mathrm{A}, \text { bulk }}\left(\Gamma_{\text {max }}-\Gamma_{\mathrm{B}}\right) \exp \left(-g^{\ddagger} \theta_{\mathrm{B}}\right)=k_{o x}^{\prime \prime} e^{\frac{(1-\alpha) F}{R T} E} \Gamma_{\mathrm{B}} \exp \left(\left(g-g^{\ddagger}\right) \theta_{\mathrm{B}}\right)$

Rearrangement gives the applied potential $E$

$$
E=\frac{R T}{F}\left(\ln \left(\frac{k_{r e d}^{\prime \prime} c^{0}}{k_{o x}^{\prime \prime}}\right)+\ln \left(\frac{c_{\mathrm{A}, b u l k}}{c^{0}}\right)+\ln \left(\frac{\left(\Gamma_{\max }-\Gamma_{\mathrm{B}}\right)}{\Gamma_{\mathrm{B}}}\right)-g \theta_{\mathrm{B}}\right)
$$

Equation 14 can be expressed in terms of the concentration of $A$ and surface coverage of $B$ yielding an expression for the formal potential $E_{f}^{0}$

$E=E_{f}^{0}+\frac{R T}{F} \ln \left(\frac{c_{\mathrm{A}, b u l k}}{c^{0}}\right)+\frac{R T}{F} \ln \left(\frac{\left(\Gamma_{\max }-\Gamma_{\mathrm{B}}\right)}{\Gamma_{\mathrm{B}}}\right), \quad$ where $E_{f}^{0}=\frac{R T}{F}\left(\ln \left(\frac{k_{r e d}^{\prime \prime} c^{0}}{k_{o x}^{\prime \prime}}\right)-g / 2\right) \quad$ (Eq. 15)

Note that because $k_{r e d}^{\prime \prime}\left(\mathrm{m}^{3} \mathrm{~mol}^{-1} \mathrm{~s}^{-1}\right)$ and $k_{o x}^{\prime \prime}\left(\mathrm{s}^{-1}\right)$ have different units, the standard (unity) concentration $c^{0}\left(\mathrm{~mol} \mathrm{~m}^{-3}\right)$ is introduced to ensure unitless quantities of which to take logarithms. It becomes apparent that the standard formal potential $E_{f}^{0}$, for a single-step electrodeposition reaction mechanism, is most suitably defined as the potential at which the surface coverage $\Gamma_{\mathrm{B}}$ at equilibrium is equal to half the maximum coverage in the presence of a standard concentration of the reactant. 
Note that $E_{f}^{0}$ is mathematically ill-defined if $\Gamma_{\mathrm{B}}$ defined at equilibrium equals either a monolayer coverage, $\Gamma_{\max }$, or zero.

\subsection{Mathematical model}

Considering the above, we next develop a mathematical model for the electro-sorption reaction shown in Equation 1 occurring at a macroelectrode. In the absence of natural convection and with sufficient inert supporting electrolyte, the mass transport of species $\mathrm{A}(a q)$ diffusing from semiinfinite solution to a macro-electrode is described by Fick's second law of diffusion in one-dimension

$$
\text { all } x, \quad \frac{\partial c_{\mathrm{A}}}{\partial t}=D_{\mathrm{A}} \frac{\partial^{2} c_{\mathrm{A}}}{\partial x^{2}}
$$

where $x$ is the distance from the electrode $(\mathrm{m})$. At the electrode interface, $x=0$, the rate of the heterogeneous reaction is given by Equation 9

$x=0, \quad D_{\mathrm{A}}\left(\frac{\partial c_{\mathrm{A}}}{\partial x}\right)_{x=0}=k_{r e d}^{\prime} c_{\mathrm{A}, 0}\left(\Gamma_{\max }-\Gamma_{\mathrm{B}}\right) \exp \left(-g^{\ddagger} \theta_{\mathrm{B}}\right)-k_{o x}^{\prime} \Gamma_{\mathrm{B}} \exp \left(\left(g-g^{\ddagger}\right) \theta_{\mathrm{B}}\right)$

The electro-sorption rate constants $k_{\text {red /ox }}^{\prime}$ can be expressed in terms of an applied potential $E$, relative to the standard formal potential $E_{f}^{0}$, and a standard electro-sorption rate constant $k_{0}^{\prime}\left(\mathrm{m}^{3} \mathrm{~mol}^{-}\right.$ $\left.{ }^{1} s^{-1}\right)$

$$
\begin{gathered}
k_{r e d}^{\prime}=k_{0}^{\prime} e^{-\frac{\alpha F}{R T}\left(E-E_{f}^{0}\right)} \\
k_{o x}^{\prime}=k_{0}^{\prime} c^{0} e^{-g / 2} e^{\frac{(1-\alpha) F}{R T}\left(E-E_{f}^{0}\right)}
\end{gathered}
$$

The term $c^{0} e^{-g / 2}$ arise from the incorporation of $E_{f}^{0}$ defined in Section 2.2, see Supporting Information (SI) Section 1 for more detail. For conservation of mass, the rate of change in surfacebound species $B$ is equal to the rate of depletion of $A$ the interface

$$
\operatorname{flux} j=-D_{\mathrm{A}}\left(\frac{\partial c_{\mathrm{A}}}{\partial x}\right)_{x=0}=-\frac{\partial \Gamma_{B}}{\partial t}
$$


In a cyclic voltammetry simulation, initially, the concentration of species $\mathrm{A}$ is uniform throughout the solution with a zero coverage of B present

$$
t=0,0 \leq x \leq x_{\max }\left\{\begin{array}{c}
c_{\mathrm{A}, x}=1 \\
\Gamma_{\mathrm{B}}=0
\end{array}\right.
$$

The potential applied to the electrode $E$ varies linearly with time from a starting potential $E_{\text {initial }}$

$$
E=E_{\text {initial }}-v t
$$

$v$ is the scan rate $\left(\mathrm{V} \mathrm{s}^{-1}\right)$. At time $t=t_{\text {switch }}$, the potential reaches $E_{\text {switch }}$ and the potential sweep changes direction

$$
E=E_{\text {switch }}+v\left(t-t_{\text {switch }}\right) \quad(\text { Eq.23) }
$$

The current obtained during the voltammetric sweep is directly related to the flux $j$ at the electrode

$$
I=-F A j \quad(\text { Eq.24) }
$$

where $I$ is current (A) and $A$ is the geometric area of the electrode $\left(\mathrm{m}^{2}\right)$.

\subsection{Dimensionless Parameters}

The definitions of the dimensionless parameters employed are given in Table 1 and are used to report the simulation results. The advantages in using dimensionless parameters as opposed to dimensional parameters is that; first, the number of independent parameters is reduced to a minimum and consequently, second, the number of simulations required to describe a wide range of experimental conditions is reduced.

Table 1. Definition of dimensionless parameters; where $r_{e}$ is the radius of the disc electrode $(m), D_{\mathrm{A}}$ is diffusion coefficient of species A $\left(\mathrm{m}^{2} \mathrm{~s}^{-1}\right)$ and $\Gamma_{\max }$ is the maximum surface coverage $\left(\mathrm{mol} \mathrm{m}^{-2}\right)$.

\begin{tabular}{ccc}
\hline Dimensionless parameters & & Normalisation \\
\hline$T$ & Time & $D_{\mathrm{A}} t / r_{e}{ }^{2}$
\end{tabular}




\begin{tabular}{|c|c|c|}
\hline$X$ & spatial coordinates & $x / r_{e}$ \\
\hline$C_{\mathrm{J}}$ & concentration of species J & $c_{\mathrm{J}} / c_{\mathrm{A}, \text { bulk }}$ \\
\hline$\theta_{\mathrm{B}}$ & fractional surface coverage of species B & $\Gamma_{\mathrm{B}} / \Gamma_{\max }$ \\
\hline$d_{\mathrm{J}}$ & diffusion coefficient of species J & $D_{\mathrm{J}} / D_{\mathrm{A}}$ \\
\hline$\sigma$ & scan rate & $\left(r_{e}^{2} / D_{\mathrm{A}}\right)(F / R T) v$ \\
\hline$B$ & saturation parameter & $\frac{r_{e} c_{\mathrm{A}, \text { bulk }}}{\Gamma_{\max }}$ \\
\hline$\Theta$ & potential & $\left(\frac{F}{R T}\right)\left(E-E_{f}^{0}\right)$ \\
\hline$K_{0}^{\prime}$ & $\begin{array}{l}\text { The standard electro-sorption rate } \\
\text { constant }\end{array}$ & $k_{0}^{\prime} \Gamma_{\max } r_{e} / D_{\mathrm{A}}$ \\
\hline$K_{\text {red }}^{\prime}$ & Electro-adsorption rate constant & $k_{\text {red }}^{\prime} \Gamma_{\max } r_{e} / D_{\mathrm{A}}$ \\
\hline$K_{o x}^{\prime}$ & Electro-desorption rate constant & $k_{o x}^{\prime} \Gamma_{\max } r_{e} / D_{\mathrm{A}} c_{\mathrm{A}, \text { bulk }}$ \\
\hline$J$ & flux & $\frac{I}{\pi r_{e} F D_{\mathrm{A}} c_{\mathrm{A}, b u l k}}$ \\
\hline
\end{tabular}

The dimensionless diffusion equation becomes

$$
0<X<X_{\max } \text { and } 0<T<T_{\max } \quad\left\{\left(\frac{\partial C_{\mathrm{A}}}{\partial T}\right)_{X}=d_{\mathrm{A}} \frac{\partial^{2} C_{\mathrm{A}}}{\partial X^{2}}\right.
$$

The boundary conditions of the model in the dimensionless form:

$$
\begin{gathered}
0<T<T_{\max } \\
X=0
\end{gathered}\left\{\begin{array}{c}
d_{\mathrm{A}}\left(\frac{\partial C_{\mathrm{A}}}{\partial X}\right)_{X=0}=K_{r e d}^{\prime} C_{\mathrm{A}, 0}\left(1-\theta_{\mathrm{A}}\right) e^{-g^{\ddagger} \theta_{\mathrm{B}}}-K_{o x}^{\prime} \theta_{\mathrm{B}} e^{\left(g-g^{\ddagger}\right) \theta_{\mathrm{B}}} \\
F l u x J=-d_{\mathrm{A}}\left(\frac{\partial C_{\mathrm{A}}}{\partial X}\right)_{X=0}=-\frac{1}{B} \frac{\partial \theta_{\mathrm{B}}}{\partial T}
\end{array}\right.
$$

Here, the dimensionless electro-sorption rate constants $K_{\text {red/ox }}^{\prime}$ are equal to 


$$
K_{\text {red }}^{\prime}=K_{0}^{\prime} e^{-\alpha \Theta} \text { and } K_{o x}^{\prime}=K_{0}^{\prime} C^{0} e^{-g / 2} e^{(1-\alpha) \Theta}
$$

where $\Theta$ is the dimensionless applied potential relative to the formal potential $E_{f}^{0}$

$$
\Theta=\frac{F}{R T}\left(E-E_{f}^{0}\right)
$$

At the start of the cyclic voltammetry simulation

$$
T=0 \quad \begin{cases}C_{\mathrm{A}, X}=1 & \text { for } 0 \leq X \leq X_{\max } \\ & \theta_{\mathrm{B}}=0\end{cases}
$$

During the simulation, the dimensionless applied potential $\Theta$ varies linearly with dimensionless time $T$ and dimensionless scan rate $\sigma$

$$
\Theta=\Theta_{\text {initial }}-\sigma T
$$

When $T=T_{\text {switch }}$, the sweep direction is reversed

$$
\Theta=\Theta_{\text {switch }}+\sigma\left(T-T_{\text {switch }}\right) \quad \text { (Eq. 31) }
$$

The dimensionless flux $J$ at the electrode surface is

$$
J=-d_{\mathrm{A}}\left(\frac{\partial C_{\mathrm{A}}}{\partial X}\right)_{X=0}
$$

The dimensionless flux $J$ can convert back to dimensional current $(A)$ for a macro-disc electrode via the equation

$$
I=\pi r_{e} F D_{\mathrm{A}} c_{\mathrm{A}, \text { bulk }} J
$$

\subsection{Numerical simulation and convergence}

The diffusion equation for the one-dimensional simulation was discretised using the Backward Implicit Method ${ }^{72-73}$ with a uniform temporal grid and a spatial grid that expands exponentially ${ }^{74}$ from the electrode surface $X_{0}$ to $X_{\max }$ 


$$
\begin{gathered}
X_{i}=\left\{\begin{array}{c}
X_{0}=0 \\
X_{i}=X_{i-1}+h * \exp (i \omega), \quad X<X_{\text {max }} \\
X_{i}=X_{\text {max }}, X \geq X_{\text {max }}
\end{array}\right. \\
X_{\text {max }}=6 \sqrt{T_{\text {max }}}
\end{gathered}
$$

where $h$ is the initial grid spacing, $\omega$ is the expansion factor and $X_{\max }$ is the extent of the dimensionless distance defined well beyond the Nernst diffusion layer ${ }^{75}$ for the duration of the simulation $T_{\max }{ }^{74,76}$ The matrix containing the sets of discretised diffusion equations, including the non-linear term for the heterogeneous reaction, were solved via the Newton-Raphson's root-finding method using the extended Thomas algorithm. ${ }^{74}$ The values of the incremental spatial, temporal point, expansion factor and Newton-Raphson's tolerance level $(\varepsilon)$ were set to a small value to ensure numerical convergence and mass conservation for each simulation. ${ }^{77}$ The simulations were written in C++ and ran on a dual Intel (R) Core (TM) E5-2640 v4 CPU $2.40 \mathrm{GHz}$ PC with $16.00 \mathrm{~GB}$ of RAM, with a typical run time of approximately one minute. All simulation results were thoroughly checked and benchmarked with two limiting cases as described below, with more data shown in the SI Section 2 and 3 .

\section{Limiting Case One}

First, the forward electro-sorption step together with the mass transport of species A was checked; in the limiting case of a small $K_{0}^{\prime}$ leading to an irreversible deposition and a sufficiently large surface coverage, such that less than $1 \%$ of the sites are occupied, the resulting peak flux matches with that predicted by the irreversible Randles- Ševčík equation ${ }^{78-79}$ with an excellent agreement (SI Section 2)

$$
J_{\text {peak, limiting Case One }}=-0.496 \sqrt{\alpha \sigma} \quad \text { (Eq. 36) }
$$

where $J_{\text {peak, limiting Case one }}$ is the dimensionless peak flux. Furthermore, the dimensionless peak potential $\Theta_{\text {peak, irreversible }}$ matches that predicted for irreversible electrode kinetics (SI Section 2). ${ }^{74}$

$$
\Theta_{\text {peak, limiting Case One }}=\frac{1}{\alpha} \ln \left(\frac{K_{0}^{\prime}}{\sqrt{\alpha \sigma}}-0.78\right) \quad \text { (Eq. 37) }
$$




\section{Limiting Case Two}

Similar to Case One, the electro-desorption of the surface-bound B can be benchmarked in the limiting case of a small $K_{0}^{\prime}$. Here, for $T=0$, the initial coverage $\theta_{\mathrm{B}}$ is set to unity with the voltammetric sweep in the anodic direction to drive the oxidation of $\mathrm{B}(a d s) \rightarrow \mathrm{A}(a q)$; an analytical expression for flux $J$ can be derived when the surface interactions are ideal, $g=g^{\ddagger}=0$, giving an expression of flux $J$ as a function of dimensionless applied potential $\Theta$ (see SI Section 3 for a step-by-step derivation)

$$
J_{\text {limiting Case } T w o}=K_{0}^{\prime} e^{\frac{-K_{0}^{\prime} B}{(1-\alpha) \sigma} e^{(1-\alpha) \Theta}+(1-\alpha) \Theta}
$$

Furthermore, the dimensionless peak potential $\left(\Theta_{\text {peak, limiting case } 2}\right)$ and dimensionless peak flux $\left(J_{\text {peak, limiting case } 2}\right)$ can be obtained when the derivative $J_{\text {limiting case } 2}$, with respect to $\Theta$, equals zero

$$
\begin{aligned}
& \Theta_{\text {peak, limiting Case Two }}=\frac{1}{1-\alpha} \ln \left(\frac{(1-\alpha) \sigma}{B} \frac{1}{K_{0}^{\prime}}\right) \\
& J_{\text {peak, limiting Case } T w o}=(1-\alpha) \sigma B^{-1} e^{-1}
\end{aligned}
$$




\section{Results and Discussion}

The mathematical model discussed above describes the one-step electrosorption reaction (Equation 1) where a solution phase species $\mathrm{A}(a q)$ transfers an electron at the electrode interface to form a surface-bound product with maximum coverage of a monolayer. Specifically, Butler-Volmer kinetics are coupled with the Frumkin adsorption isotherm providing a rate expression for the heterogeneous reaction allowing non-ideal surface interactions to be studied in terms of the Frumkin interaction parameter $g$.

In the following results and discussion, we provide new physical insights on how the non-ideal surface interactions coupled with mass transport affects the current-voltage transient in a typical voltammetry experiment, in which the dynamically applied potential changes with time, notably the classical triangular potential waveform in cyclic voltammetry which will inevitably lead to mass transport induced dis-equilibrium in the vicinity of the electrode. First, however, we consider the Frumkin adsorption isotherm where both mass transport and adsorption-desorption are in equilibrium to understand the possible non-ideal surface interactions more fully and focus on the emergence of phase transition behaviour in the voltammetry for strongly attractive surfaces interactions.

\subsection{Phase Transitions Simulated for Equilibrium; No Mass Transport Limitations}

In this section, the effect of surface interaction parameter $g$ for Frumkin adsorption isotherm is discussed in the context of the electro-sorption reaction under mass transport and surface reaction equilibrium. First, a common ground between the adsorption isotherm and electro-sorption isotherm has to be established for direct comparison. Hereafter in the article, the notation of the factional surface coverage for pure adsorption-desorption processes will be unified with that used in the electro-sorption reaction (i.e. $\theta=\theta_{\mathrm{B}}$ ) to avoid possible confusion. 
The common ground that both the adsorption isotherm and the electro-sorption reaction share is a thermodynamic equilibrium position controlled by the rate of the corresponding forward and backward reactions. Here, a dimensionless equilibrium constant $K$ is introduced, allowing a direct comparison between the two equilibria. For pure adsorption-desorption, the dimensionless equilibrium constant $K$ is equal to $\frac{k_{a d s}}{k_{d e s}} c_{\mathrm{A}, b u l k}$. A similar expression for the electro-sorption reaction can be obtained when flux, either in the dimensional form (Equation 17) or the dimensionless equivalent (Equation 26), is equal to zero

$$
K=\frac{K_{r e d}^{\prime}}{K_{o x}^{\prime}}=\frac{k_{r e d}^{\prime}}{k_{o x}^{\prime}} c_{\mathrm{A}, b u l k}(\text { Eq. 41) }
$$

This leads to the expression relating the dimensionless equilibrium constant $K$ to the fractional coverage $\theta_{\mathrm{B}}$

$$
K C_{\mathrm{A}, 0}=\frac{\theta_{\mathrm{B}}}{1-\theta_{\mathrm{B}}} \exp \left(g \theta_{\mathrm{B}}\right) \quad(\text { Eq. 42) }
$$

Note that, the dimensionless interfacial concentration $C_{\mathrm{A}, 0}$ is equal to $c_{\mathrm{A}, 0} / c_{\mathrm{A}, \text { bulk }}$; in the case of the system at equilibrium, $c_{\mathrm{A}, 0}$ equals $c_{\mathrm{A}, b u l k}$ and thus $C_{\mathrm{A}, 0}$ is unity. We leave the notation $C_{\mathrm{A}, 0}$ as is in this section.

Figure 1 shows a plot of $\theta_{\mathrm{B}}$, equilibrium against $K C_{\mathrm{A}, 0}$, for different Frumkin interaction parameter $g$. Solid lines show the prediction from Frumkin adsorption isotherm and circles are results from the electro-sorption simulation when the system is at equilibrium. For all cases of $g$, whether attractive, ideal or repulsive adsorbate-adsorbate interactions, an increase in $K C_{\mathrm{A}, 0}$ leads to an increase in the fractional coverage of $\theta_{\mathrm{B}}$ at equilibrium. Excellent agreements are seen between the simulation results and the Frumkin adsorption isotherm for all values of $K C_{\mathrm{A}, 0}$ over the full range of $g$ studied. As $g$ becomes increasingly negative, the surface interaction becomes increasingly attractive and a lower $K C_{\mathrm{A}, 0}$ is required to reach half fractional coverage as indicated by the dashed line. 
The physical meaning of $K C_{\mathrm{A}, 0}$ varying along the $\mathrm{x}$-axis can be interpreted with experimental conditions; under system equilibrium, the dimensionless concentration at the electrode interface $C_{\mathrm{A}, 0}$ is trivially unity thus it is $K$ that changes along the $\mathrm{x}$-axis. From the definition of $K$ for adsorption isotherms, this is a change in the dimensional bulk concentration $\left(c_{\mathrm{A}, \text { bulk }}\right)$ since the ratio of $k_{a d s} / k_{\text {des }}$ is a constant "given by the universe". For the electro-sorption reaction, however, $K$ can be changed either by $c_{\mathrm{A}, \text { bulk }}$ and/or the applied potential (Equation 18 and 19).

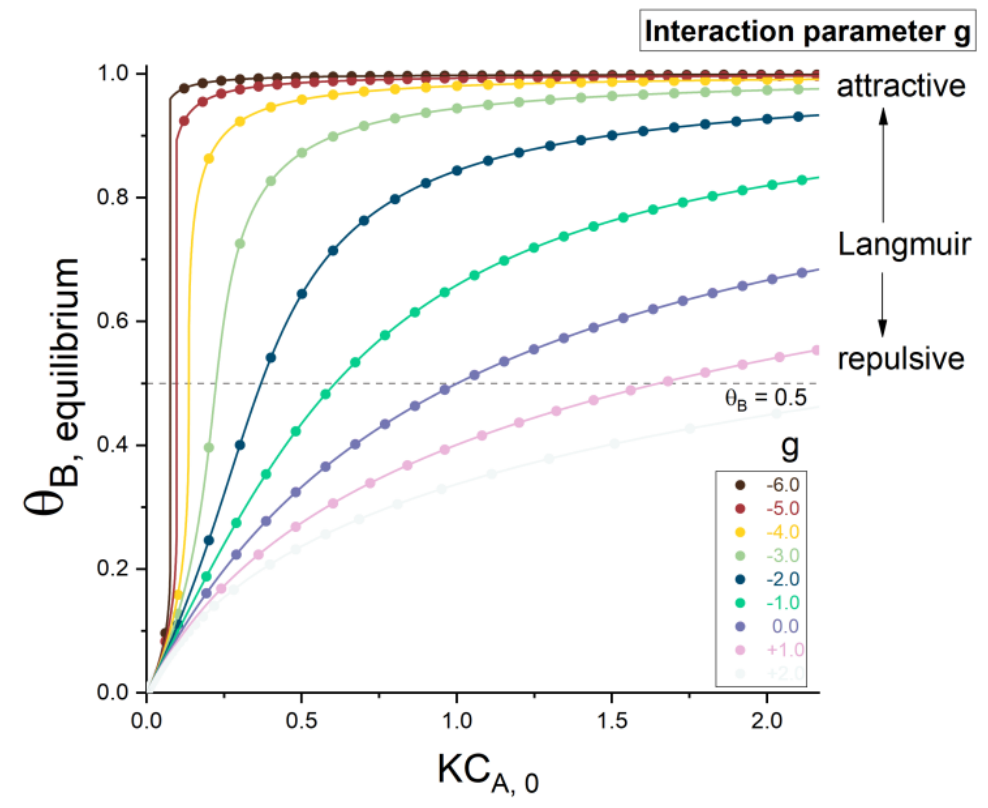

Figure 1. Plot of $\theta_{\mathrm{B}, \text { equilibrium }} v \mathrm{~K} \mathrm{C}_{\mathrm{A}, 0}$ from the Frumkin adsorption isotherm for a range of interaction parameter $g$ (solid lines). Circles: simulation results obtained by setting $C_{\mathrm{A}}$ over all space to unity until equilibrium are established with no more than $10^{-7} \%$ change in the fractional coverage from the last iteration.

Figure 2 shows the equivalent plot of Figure 1 but with normalisation in the $\mathrm{x}$-axis to the value of $K C_{\mathrm{A}, 0}$ leading to half fractional coverage at equilibrium $\left(K C_{\mathrm{A}, 0} / K_{\theta_{\mathrm{B}}=0.5}\right)$. Substitution of $C_{\mathrm{A}, 0}=C_{\mathrm{A}, b u l k}$ and $\theta_{\mathrm{B}}=0.5$ into Equation 42 gives

$$
K_{\theta_{\mathrm{B}}=0.5} C_{\mathrm{A}, \text { bulk }}=\exp \left(\frac{g}{2}\right)
$$

This normalisation standardises the plots of all $g$ in Figure 2 resulting in an 'isosbestic point' like behaviour when $K C_{\mathrm{A}, 0} / K_{\theta_{\mathrm{B}}=0.5}$ equals unity. It now becomes apparent that, as the interactions between the surface-bound species become increasingly attractive (negative $g$ ), the $\theta_{\mathrm{B}}$ vs $K C_{\mathrm{A}, 0} / K_{\theta_{\mathrm{B}}=0.5}$ transient becomes more and more sigmoidal. When $g$ approaches -4.0 (yellow line), 
a sudden and abrupt change in $\theta_{\mathrm{B}}$ can be seen near unity of $K C_{\mathrm{A}, 0} / K_{\theta_{\mathrm{B}}=0.5}$ indicating a "phase transition like" behaviour. When $g$ is more negative than -4.0 , a counter-intuitive 'S-shape' transient about $\theta_{\mathrm{B}}=0.5$ and $K C_{\mathrm{A}, 0} / K_{\theta_{\mathrm{B}}=0.5}=1$ can be seen. From this one infers that there is a sudden jump from a low to high coverage as shown by the dashed line in Figure 2a) since physically an increasing $\frac{K C_{\mathrm{A}, 0}}{K_{\theta_{\mathrm{B}}=0.5}}$ cannot 'fall back' on itself. Therefore, the region where the curve changes direction with respect to the $\mathrm{x}$-axis can be thus viewed as "forbidden". The above phenomenon is next simulated using the developed simulation model for the values of $g$ discussed. The results are overlaid in Figure 2.
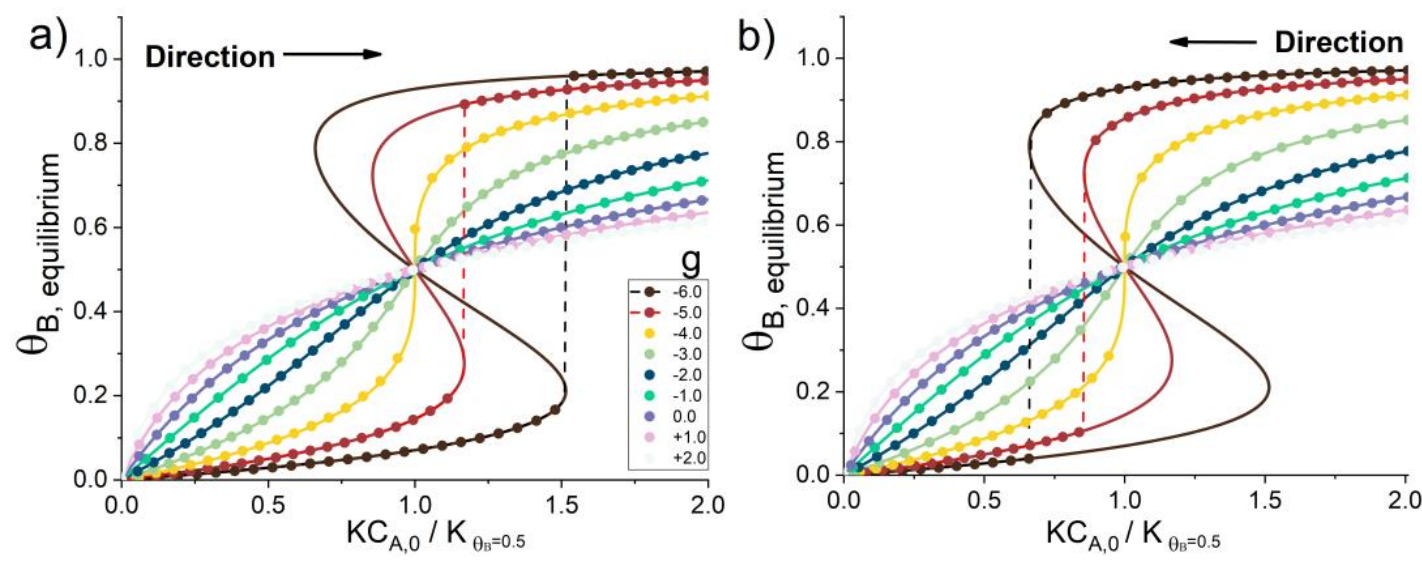

Figure 2. Plot of $\theta_{\mathrm{B}, \text { equilibrium }}$ vs $K C_{\mathrm{A}, 0} / K_{\theta_{\mathrm{B}}=0.5}$ for different values of interaction parameter $g$. The solid line shows the analytical Frumkin adsorption isotherm. The overlaid circles are the simulation results with either (a) an incremental increase in $K C_{\mathrm{A}, 0} / K_{\theta_{\mathrm{B}}=0.5}$ from zero or (b) incremental decrease in $K C_{\mathrm{A}, 0} / K_{\theta_{\mathrm{B}}=0.5}$ from 2.0. The dashed lines infer the sudden jump in coverage of $\mathrm{B}$.

To mimic the equilibration between the solution and the electrode layer in the simulation, the concentration of species $\mathrm{A}$ over the entire simulation space was set initially to $C_{\mathrm{A}, \text { bulk }}$ and the simulation allowed to iterate but with the solution concentration reset to $C_{\mathrm{A}, \text { bulk }}$ after each temporal iteration, until the fractional coverage $\theta_{\mathrm{B}}$ reaches equilibrium with the bulk solution. The equilibrium was consider established when the fractional change in the coverage of $\theta_{\mathrm{B}}$ was no more than $10^{-7} \%$ from the previous temporal iteration. Figure 2 shows $\theta_{\mathrm{B}}$ as a function of $K C_{\mathrm{A}, 0} / K_{\theta_{\mathrm{B}}=0.5}$ for two cases; first when $K C_{\mathrm{A}, 0} / K_{\theta_{\mathrm{B}}=0.5}$ is incrementally increased from zero and second when it is decreased from a large value corresponding to a high, near unity, coverage of B. These data were shown as circles for 
different $g$. In all cases of $g$, and both direction of changes in $C_{\mathrm{A}, \text { bulk }}$, the simulation results all overlaid on the transient as predicted by the Frumkin adsorption isotherm (solid lines). For the non-phase transition $g$ studied $(-4.0<g \leq+2.0)$, the results were independent to the direction of change in $C_{\mathrm{A}, \text { bulk }}$. When $g$ equal to -4.0 , a vertical gradient between $\theta_{\mathrm{B}, \text { equilibrium }}$ from 0.4 to 0.6 can be seen when $K C_{\mathrm{A}, 0} / K_{\theta_{\mathrm{B}}=0.5}$ approaches unity. When $g$ is more negative than -4.0 , a point of discontinuity from the sigmoidal transient is seen with $\theta_{\mathrm{B}, \text { equilibrium }}$ changing abruptly from 0.2 to 0.9 for an increase in $K C_{\mathrm{A}, 0} / K_{\theta_{\mathrm{B}}=0.5}$ and from 0.9 to 0.2 for a decreasing in $K C_{\mathrm{A}, 0} / K_{\theta_{\mathrm{B}}=0.5}$ as discussed above; note that the simulated data do not enter the 'forbidden' part of the sigmoidal curve where the direction of the analytical curve is opposite to the direction of change in $K C_{\mathrm{A}, 0} / K_{\theta_{\mathrm{B}}=0.5}$. These abrupt changes in fractional coverage signal a phase transition, and it only occurs with values of $g \leq-4.0$.

Having understood the effects of non-ideal surface interaction under the conditions of both surface kinetics and mass transport equilibrium. The focus now turns to voltammetric studies where the dynamic change in applied potential with time may not necessarily be kinetically reversible nor in mass transport equilibrium near the vicinity of the electrode.

\subsection{Cyclic Voltammetry: Non-ideal Leading to Phase Transition}

This section aims to provide new physicochemical insights on how the non-ideal adsorbate-adsorbate interaction, $g$, affects the cyclic voltammograms of the electro-sorption reaction under planar diffusion conditions. First, the influence of $g$ on voltammograms is studied for the case of reversible electro-sorption kinetics $\left(K_{0}^{\prime}\right)$. Second, for each value of $g$, the effects of the $K_{0}^{\prime}$ (extent of reversibility), are studied. We then briefly investigate the effect of $g^{\ddagger}$, the activated complex (transition state) adsorbate interaction, for different values of $g$. Then, we turn to investigate the effects of mass transport on the cyclic voltammograms and, in particular, for the case where a critical value of $g$ leads to a phase transition.

Simulated voltammograms as a function of $g$ and corresponding to fast and reversible electrosorption kinetics $\left(K_{0}^{\prime}=100\right)$ are shown in Figure 3. Here, the term fast (or slow) kinetics is relative to 
the mass transport under ideal surface interactions $g=0$. Other simulation parameters are: $B=$ $100, \sigma=100, \alpha=0.5, g^{\ddagger}=0$. For all cases of $g$, a cathodic peak is seen during the forward sweep with the dimensionless flux $J$ subsequently returning to zero corresponding to full coverage of $B$ which precludes any full 'diffusional tail' being seen; similarly, anodic stripping peaks are seen on the reverse sweeps. During the reverse stripping sweep, the surface-bound B is driven to form $\mathrm{A}(a q)$ leading to an anodic stripping peak after which the flux returns to zero when $\mathrm{B}(\mathrm{ads})$ becomes fully depleted. In the ideal case where the adsorbate-adsorbate surface interactions ( $g$ ) equals to zero, the voltammogram is symmetric resembling a surface-bound redox process, $\mathrm{A}($ ads $)+e^{-} \rightleftharpoons \mathrm{B}($ ads $)$ under fast electrode kinetics due to the excess of $\mathrm{A}(a q)$ at the electrode surface in comparison with the number of sites available. If the interactions on the surface become repulsive $(g>0)$, the voltammogram appears 'flattened' compare to the ideal case because a higher over-potential is required to drive the forward deposition reaction as the repulsive interaction on the surface scales with coverage. On the other hand, the voltammetric wave-shape becomes sharper and narrower for attractive surface interactions $(g<0)$. This is due to the attractive surface interaction, scaling with surface coverage, providing an additional driving force for the deposition. Furthermore, as $g$ approaches to the critical phase transition values of -4.0 or beyond, the peak current surges to a large value once the reaction has begun, as shown by the 'zoom-out' plot in the inlay of Figure 3 . Furthermore, the peak-to-peak separation increases when $g$ is beyond the critical value of -4.0 indicating an increase in the degree of irreversibility at $K_{0}^{\prime}=100$, of which more is discussed below. 


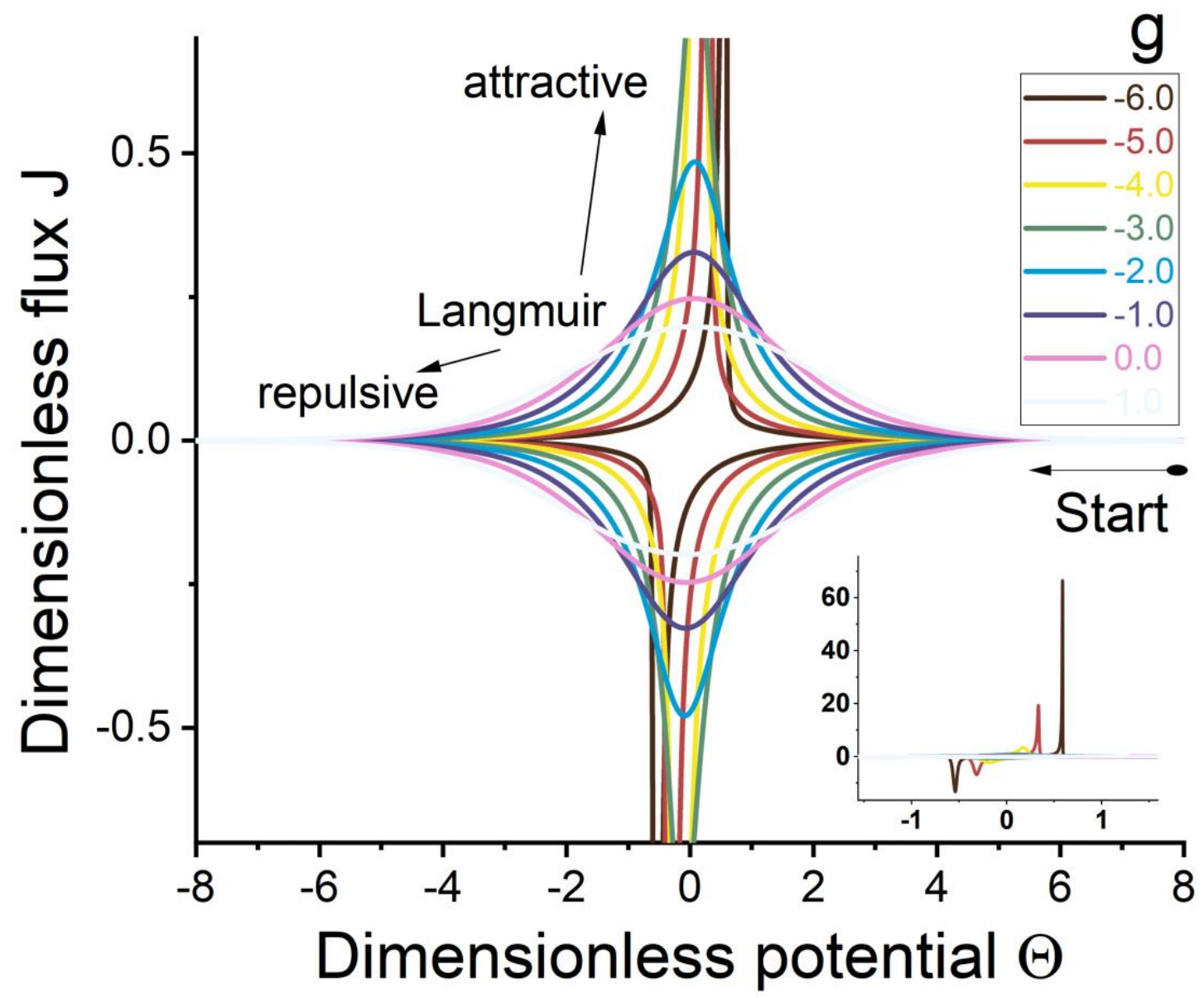

Figure 3) Simulated voltammograms as a function of surface interaction parameter $g$ under reversible electrode kinetics. The values of $g$ are shown in the figure. Other simulation parameters: $K_{0}^{\prime}=100, B=100, \sigma=100, \alpha=0.5$ and $g^{\ddagger}=0$.

Figure 4 shows a series of simulated voltammograms varying dimensionless standard electro-sorption rate constant $K_{0}^{\prime}$, from $10^{6}$ to $10^{-3}$, for a range of $g$ values. The other simulation parameters are the same as in Figure $3\left(B=100, \sigma=100, \alpha=0.5, g^{\ddagger}=0\right)$. The general feature seen across all values of $g$ is an increase in peak-to-peak separation $\Delta \Theta_{p p}\left(\Theta_{\text {anodic peak }}-\Theta_{\text {cathodic peak }}\right)$ as $K_{0}^{\prime}$ decreases. Changes in the cathodic and anodic peak fluxes are also seen but are different for attractive and repulsive surface interaction. In the case when the surface interactions are ideal, $g$ equals zero, both the anodic and cathodic peak flux decrease asymptotically to a constant value as $K_{0}^{\prime}$ decreases to a fully irreversible limit at $10^{-3}$. For repulsive surface interaction, $g$ equal to +2.0 , the cathodic peak flux increases as $K_{0}^{\prime}$ approaches $10^{-3}$ whilst anodic peak flux decreases. On the other hand, for attractive surface interactions $(g=-2.0)$ the magnitude of the cathodic peak flux is more than halved as the 
electrode kinetic turns fully irreversible; this phenomenon is amplified as $g$ decreases to -4.0 and -5.0 .
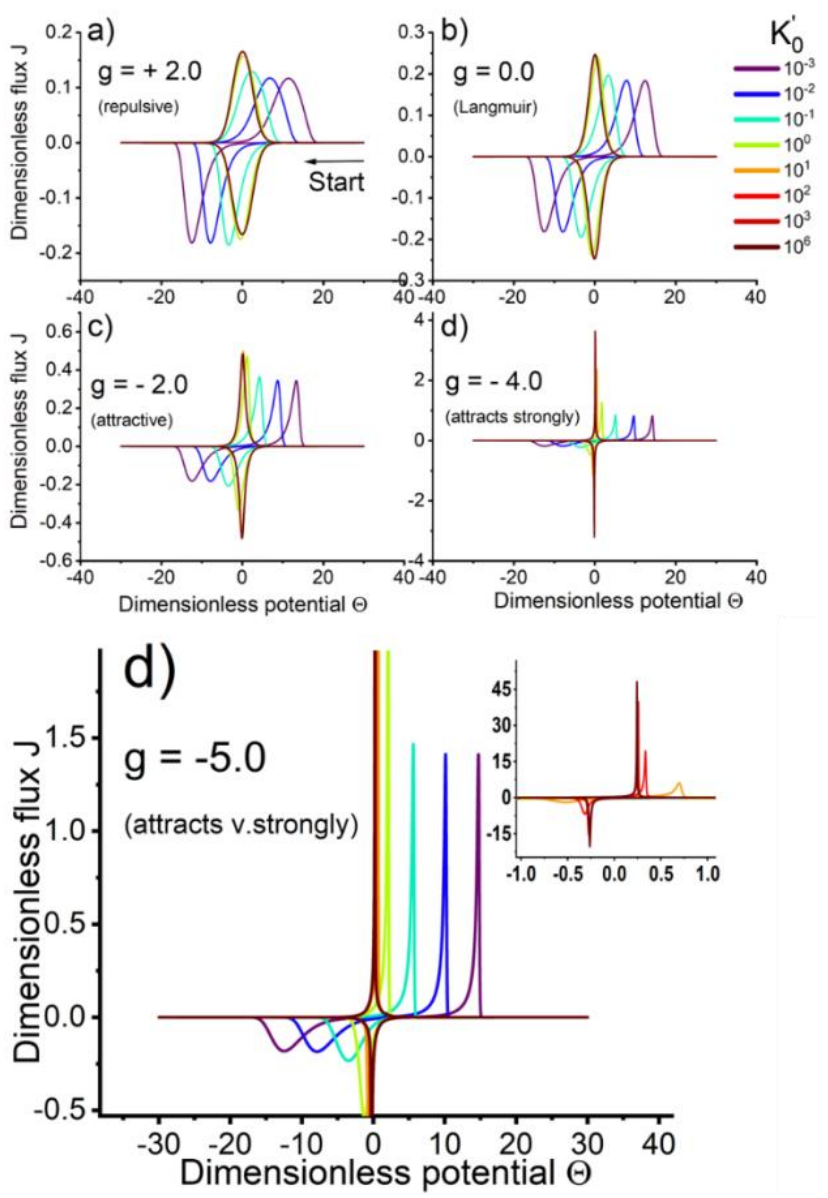

Figure 4) Simulated voltammograms as a function $K_{0}^{\prime}$ ranging from $10^{-3}$ to $10^{6}$ for different values of $g$. The values of $g$ and $K_{0}^{\prime}$ can be found in the figure. Other simulation parameters: $B=100, \sigma=100, \alpha=0.5$ and $g^{\ddagger}=0$.

An interesting observation can be seen in Figure 4 , at $g=0$, notably that full electrode reversibility was achieved at $K_{0}^{\prime}=10^{1}$ as higher orders of magnitude of $K_{0}^{\prime}$ result in identical voltammograms. In comparison for $g=-5.0$, the magnitude of peak flux at $K_{0}^{\prime}=10^{1}$ is ca one order below that seen at $K_{0}^{\prime}=10^{6}$ (inlay of Figure $4 \mathrm{~d}$ ). It is evident that a higher threshold of $K_{0}^{\prime}$ is necessary to reach the limit of full electrode reversibility as $g$ becomes more negative, particularly when $g$ approaches the critical value for a phase transition. This is as expected as the electrode reversibility reflects the interplay between surface kinetics and mass transport in the solution phase, ${ }^{80-81}$ thus indicating that the changes in the interfacial concentration of species A 'outrun' their replenishment by mass transport at negative $g$. To evidence this and to investigate the possibility of phase transition occurring under 
voltammetry conditions when $g \leq-4.0$, we next utilise the numerical simulation to provide further insight to the underlying physicochemical processes giving rise to the sharp voltammetric peaks seen in Figure 4.

Figure 5 shows the fractional surface coverage of $B$ and the corresponding concentration profiles of $A$ for the voltammograms seen in Figure 4. Figure 5 a) plots the surface coverages of B for different surface interactions $(g)$ simulated for irreversible electrode kinetics $\left(K_{0}^{\prime}=10^{-2}\right)$. The case for reversible electrode kinetics $\left(K_{0}^{\prime}=10^{6}\right)$ is shown in Figure $5 \mathrm{c}$ ). The $\mathrm{x}$-axis is time normalised over the timescale of the simulation. In the case of irreversible electrode kinetics, the surface coverage during the forward sweep $\left(\frac{T}{T_{\max }}<0.5\right)$ are relatively insensitive to the surface interaction parameter $g$ as over-potential is required to drive the sluggish reaction to overcome the effect of repulsive interaction; in contrast, under reversible electrode kinetics, the surface interaction $g$ has a rather profound impact on the surface coverage of B during the forward sweep. As $g$ becomes increasingly negative, a rapid increase in fractional coverage is seen due to the attractive adsorbate-adsorbate interactions. Under reversible electrode kinetics and with $g$ satisfying phase transition $(\leq-4.0)$ the slope of $\theta_{\mathrm{B}}$ versus $T$ is vertical as can be seen in the inlays of Figure $5 \mathrm{c}$ ).

Figures $5 \mathrm{~b}$ ) and d) show the concentration profiles of A simulated for $g=-5.0$ under irreversible and reversible electrode kinetics, respectively. As can be seen, in both cases, the interfacial concentration does not equal to the bulk concentration once the interfacial reaction is driven by the triangular potential waveform; for reversible electrode kinetics, Figure $5 \mathrm{~d}$ ), a sharp drop in the interfacial concentration, $C_{\mathrm{A}, 0}$, is seen around $\frac{T}{T_{\max }}=0.2$ compared to that seen with irreversible electrode kinetics (Figure 5b). This, together with a vertical gradient of the surface coverage, indicate that reversible electrode kinetics are essential in capturing signs of a phase transition on the time scale of a typical voltammetric experiment. 

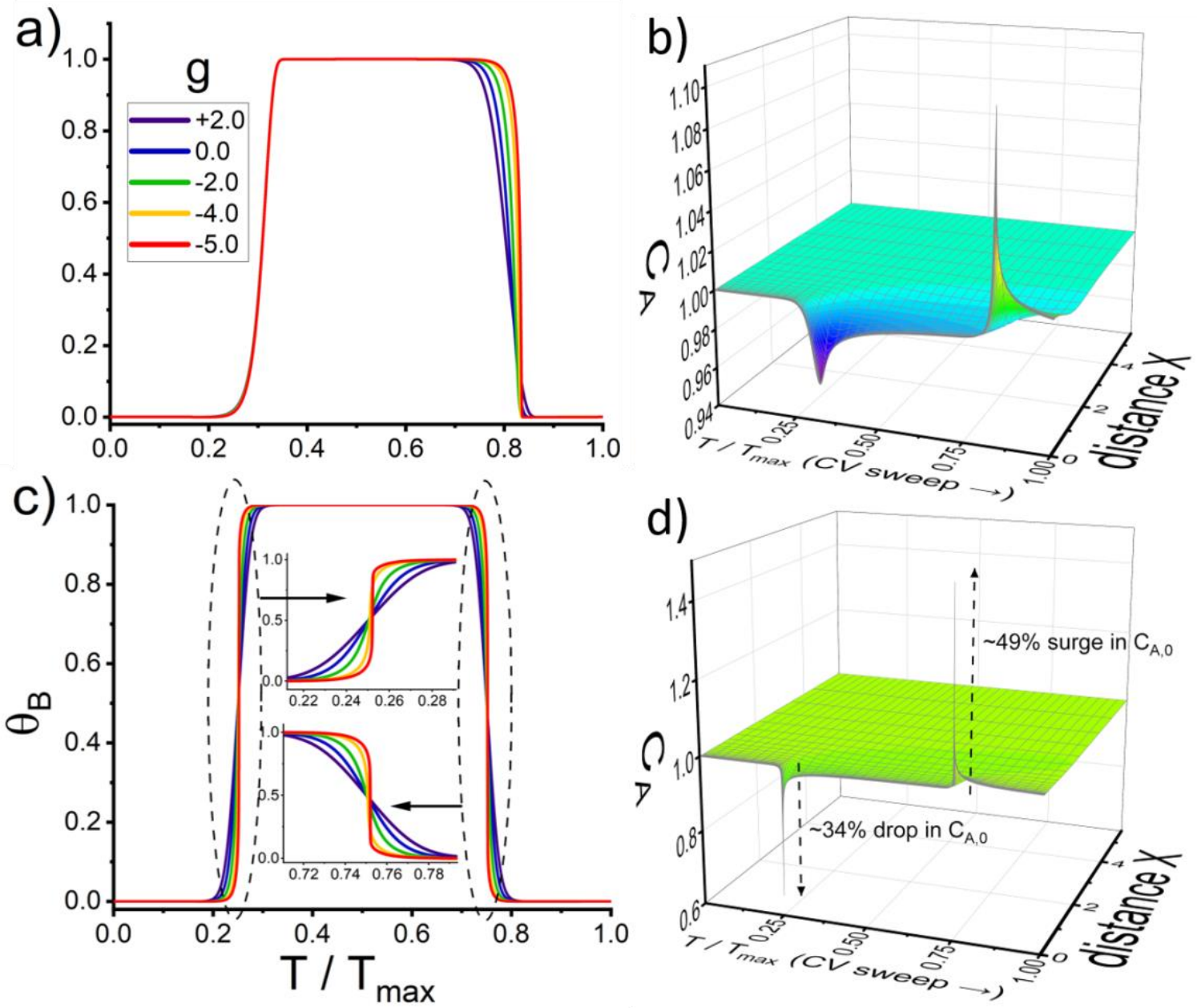

Figure 5) A selection of surface coverages and concentration profiles in above simulated cyclic voltammograms, as seen in Figure 4, are plotted. Surface coverages as a function of $\mathrm{g}:$ a) irreversible electrode kinetics, $K_{0}^{\prime}=10^{-2}$; c) reversible electrode kinetics, $K_{0}^{\prime}=10^{6}$. Concentration profile of $\mathrm{A}(a q)$ for $\left.g=-5.0: b\right)$ irreversible electrode kinetics, $\left.K_{0}^{\prime}=10^{-2} ; d\right)$ reversible electrode kinetics; $K_{0}^{\prime}=10^{6}$. Other simulation parameters: $B=100, \sigma=100, \alpha=0.5$ and $g^{\ddagger}=0$.

An important question to pose is, how does this abrupt change in $\theta_{\mathrm{B}}$ and $C_{\mathrm{A}, 0}$ seen during the voltammetric timescale relate to the plot of Frumkin adsorption isotherm in Figure 2? And, to what extent does un-equilibrated mass transport during the voltammetry affect the phase transition? To shed light on those questions underpinning the physicochemical processes occurring during the voltammetry timescale, we show the same data for the Frumkin adsorption isotherm as in Figure 6. The $x$ - and $y$-axes remain the same as Figure $2, \theta_{B} v s \frac{K C_{\mathrm{A}, 0}}{K_{\theta_{\mathrm{B}}=0.5}}$. In the voltammetric experiments, the triangular potential waveform drives the interfacial electro-sorption reaction leading a perturbation of concentration of $\mathrm{A}(a q)$ near the vicinity of the electrode, which is evident in the concentration profiles shown in Figure 5 . We have established that Equation 42 involving the dimensionless 
equilibrium constant $K$ are general for both the electro-sorption reaction and Frumkin adsorption isotherm. For Figure 6, the electro-sorption equilibrium constant $K$ is potential dependant with the interfacial concentration $C_{\mathrm{A}, 0}$ and the coverage $\theta_{\mathrm{B}}$ known from the numerical simulation. Overlaid in Figure 6 are the numerical data from voltammograms seen Figure 4 , for (a) $K_{0}^{\prime}=10^{-2}$ corresponding to irreversible and (b) $K_{0}^{\prime}=10^{6}$ for reversible electrode kinetics. The circles are data obtained during the forward sweep, and the triangles are data from the reverse sweep of the simulated voltammetry. The direction of travel for the forward sweep is positive on the x-axis $\left(K \propto \mathrm{e}^{-\Theta}, \Theta_{\text {initial }}=+30\right)$ whereas that for the reverse sweep is negative on the $\mathrm{x}$-axis $\left(\Theta_{\text {switch }}=-30\right)$. For irreversible electrode kinetics, Figure 6 a), the reaction at the surface is sluggish and it is unresponsive to the instantaneous changes in potential leading to a 'disconnection' from the Frumkin isotherm for all cases of $g$. In the case where electrode kinetics is fast and reversible, Figure $6 \mathrm{~b}$ ), matches with the Frumkin adsorption isotherm with an excellent agreement for all values of $g$ and both forward and backward sweeps. Interestingly, the cases when the interaction parameter $g$ is beyond the critical value for phase transition, i.e. $g<-4.0$, the surface $\theta_{\mathrm{B}}$ transits into the 'forbidden' region of the Frumkin adsorption isotherm. The latter is not due to $K(\Theta)$ since the dimensionless potential $\Theta$ is varied linearly but thus must result from a local change in concentration of $\mathrm{A}(a q)$ on the timescale of voltammetry. The concentration profile of $g=-5.0$ can be seen in Figure $5 \mathrm{~d}$ ), where a drop of approximately $34 \%$ of the interfacial concentration compare to bulk solution was observed during the forward sweep. At the same time, a positive and vertical slope of surface coverage against time is seen in Figure $4 \mathrm{c}$, red line. It can be concluded that transition into the 'forbidden zone' is a result of the non-equilibrated interfacial concentration under the dynamically changing voltammetric conditions. 

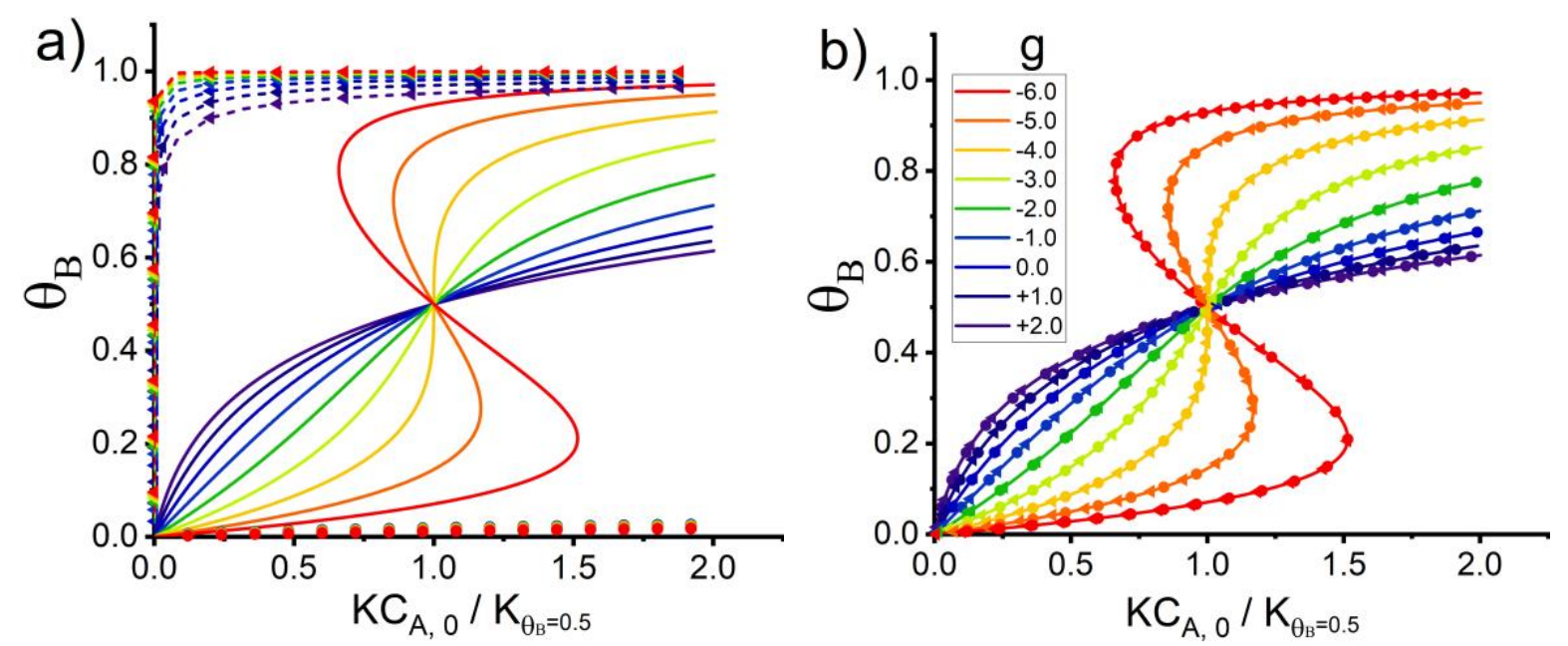

Figure 6). A plot of $\theta_{\mathrm{B}}$ against $K C_{\mathrm{A}, 0} / K_{\theta_{\mathrm{B}}=0.5}$ as a function of surface interaction parameters $g$. The numerical data obtained from the voltammograms in Figure 4 are shown; a) irreversible electrode kinetics $K_{0}^{\prime}=10^{-2}$ and b) reversible electrode kinetics $K_{0}^{\prime}=10^{-2}$. Specifically, the data from the forward sweep is shown as circles with triangles represent the backward sweep. Solid line shows the analytical prediction for the Frumkin adsorption isotherm plotted in both a) and $b$ ). The additional dashed lines in a) are used to enhance the visibility of the data for the backward sweep (triangles). The values of $g$ are shown in the figure. Other simulation parameters: $B=100, \sigma=100, \alpha=0.5$ and $g^{\ddagger}=0$.

Having understood the behaviour of $g$ and the effects that it has on voltammetry under both reversible and irreversible conditions, we next turn our attention to investigate the effect of $g^{\ddagger}$, the transition state-adsorbate interaction parameter, as a function of different values of $g$. Figure 7 shows the series of voltammograms under irreversible electron kinetics $\left(K_{0}^{\prime}=10^{-2}\right)$. The realistic values of $g^{\ddagger}$ may take any value from zero up to the value of $g$ since the transition state partially resembles that of the product on the electrode surface. For positive values of $g$ and $g^{\ddagger}$, Figure 7 a), the peak-topeak separation is increased and this can be interpreted as an increase in the activation energy for the overall reaction. For negative values of of $g^{\ddagger}$, Figure $7 \mathrm{c}$ )-e), the peak-to-peak separations decrease with an increasingly negative $g^{\ddagger}$. This is interpreted as a decrease of activation energy of the overall reaction. The values of $g^{\ddagger}$, negative nor positive, has an effect on the voltammograms under reversible electrode kinetics, shown in SI section 4. This is reasonable as the activation energy becomes unimportant when the system is thermodynamic equilibrium. 


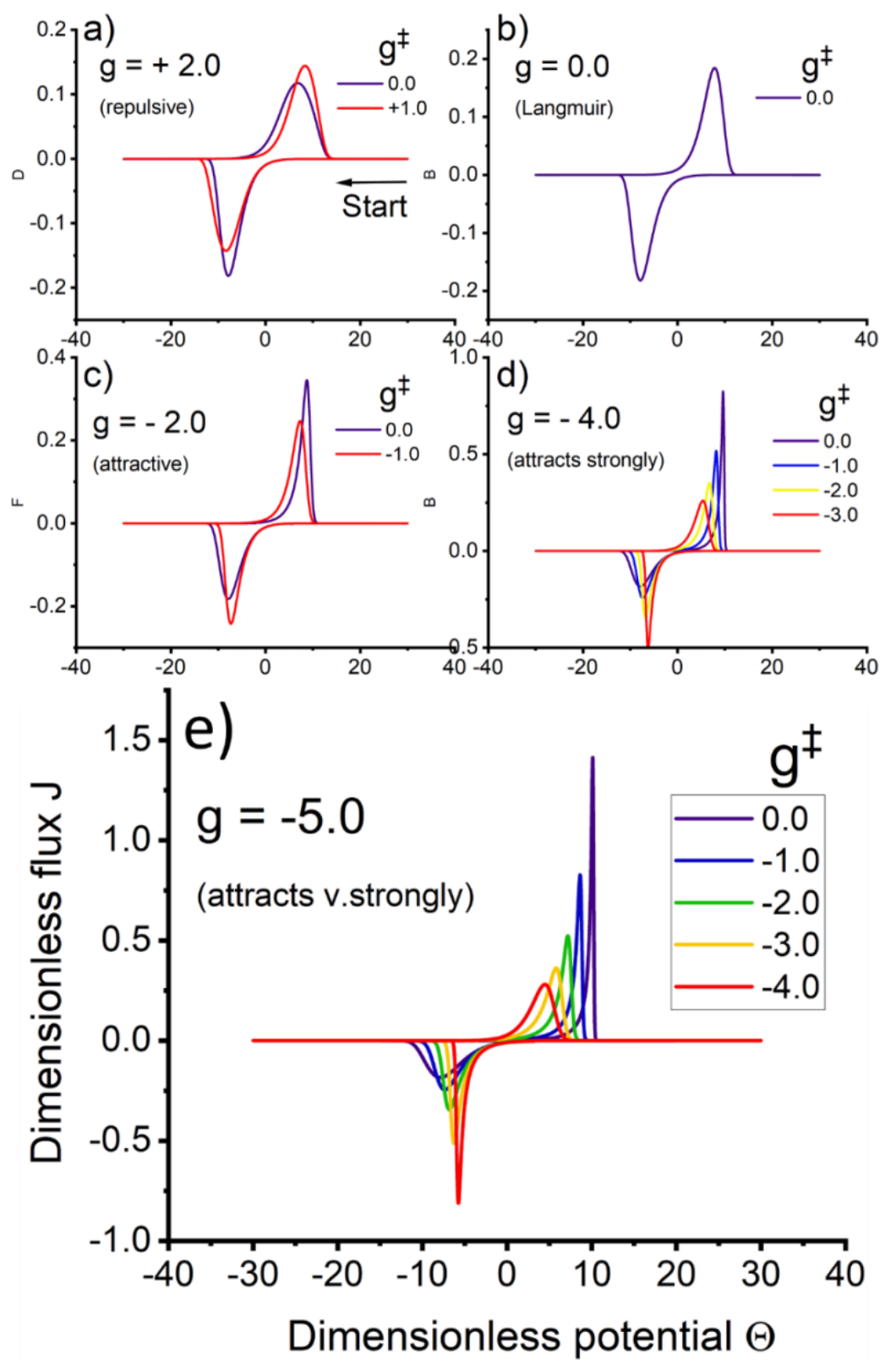

Figure 7) Series of voltammograms varying $g^{\ddagger}$ for different values of $g$. The values of $g$ and $g^{\ddagger}$ are shown in the figure. Other simulation conditions: $K_{0}^{\prime}=0.01, B=100, \sigma=10$ and $\alpha=0.5$.

In this section the independent parameters controlling the interfacial reaction, namely $K_{0}^{\prime}, g$ and $g^{\ddagger}$, have been investigated. We infer that fast and reversible electrode kinetics are essential to observe phase transition in the voltammetric timescale when the critical values of $g$ are satisfied $(\leq-4.0)$. The interfacial conditions simulated during the voltammetry induced phase transition reveal intrusions into the 'forbidden' part predicted by the Frumkin adsorption isotherm, which are otherwise inaccessible to the prediction on the assumption of mass transport equilibrium. Furthermore, we note that the transition state - adsorbate interaction $\left(g^{\ddagger}\right)$ does not affect 
voltammetry involving the behaviour of phase transition as observation of the latter requires reversible electrode kinetics. In the next section, we investigate if, and how, mass transport of the solution phase species $A$ affects voltammetry even if high concentrations of $A$ are used - in particular in the event of phase transition involving a rapid drop in the interfacial concentration is seen.

\subsection{Cyclic Voltammetry: Effect of Mass Transport}

Hitherto, the underlining physicochemical processes of the electro-sorption reaction with non-ideal surface interactions leading to phase transition were studied at a dimensionless scan rate of $\sigma=100$ and saturation parameter $B=100$, where the latter is the ratio of bulk concentration of $A$ to the total coverage (see Table 1). These parameters, for a $1 \mathrm{~mm}$ macro-disc electrode and with a typical diffusion coefficient $\left(D_{\mathrm{A}}=1 \times 10^{-9} \mathrm{~m}^{2} \mathrm{~s}^{-1}\right)$ correspond to experimental conditions of $c_{\mathrm{A}, \text { bulk }}=1 \mathrm{mM}, \Gamma_{\max }=$ $10^{-5} \mathrm{~mol} \mathrm{~m}^{-2}$ and $v \approx 3 \mathrm{mVs}^{-1}$. In literature studies, analytical expressions used to estimate $g$ from the voltammetric peak width typically assume a diffusionless system, i.e. $C_{\mathrm{A}, 0}=C_{\mathrm{A}, \text { bulk }}$ at all times, justified by the experimental use of a large concentration of the reactant $(0.1 \mathrm{M})$ with an extremely slow scan rate (often below $1 \mathrm{mVs}^{-1}$ ). ${ }^{43,45,49}$ In this section, the effect of dimensionless scan rate $\sigma$ and the saturation parameter $B$ on the resulting voltammograms will be investigated for two cases of $g$, first corresponding to no phase-transition behaviour and second for values of $g$ beyond the critical value for a phase transition.

First, the effect of the dimensionless scan rate $\sigma$ is studied for $g=-2.0$ (no phase transition) and $g=$ -5.0 (phase transition). In the electro-sorption reaction, in the limit of an excess in the interfacial concentration of A compared to the number of surface sites the voltammetric response appears to be 'surface-bound' and the flux scales linearly with $\sigma$. Figure 7 shows the simulated voltammograms under reversible electrode kinetics $\left(K_{0}^{\prime}=10^{6}\right)$ with the $y$-axis normalised to $\sigma$. As can be seen, the voltammetric shape for $g=-2.0$ (no phase transition), under reversible electrode kinetics, are relatively unperturbed over three orders of magnitude of $\sigma$; at a fast scan rate, $\sigma=1000$, the voltammetric shape starts to flatten indicating a small degree of irreversibility. For a phase transition 
value of $g=-5.0$, however, the effect of $\sigma$ is much more pronounced. The normalised peak flux $J / \sigma$ is approximately three times larger with a decrease of $\sigma$ from 1000 to 100 , and from 100 to 10 . This shows that voltammetry involving a phase transition is more sensitive to the value of $\sigma$ compared to that with no phase transition behaviour. The physicochemical reasoning leading this observation in flux is discussed below in conjunction with results from varying the saturation parameter $B$.
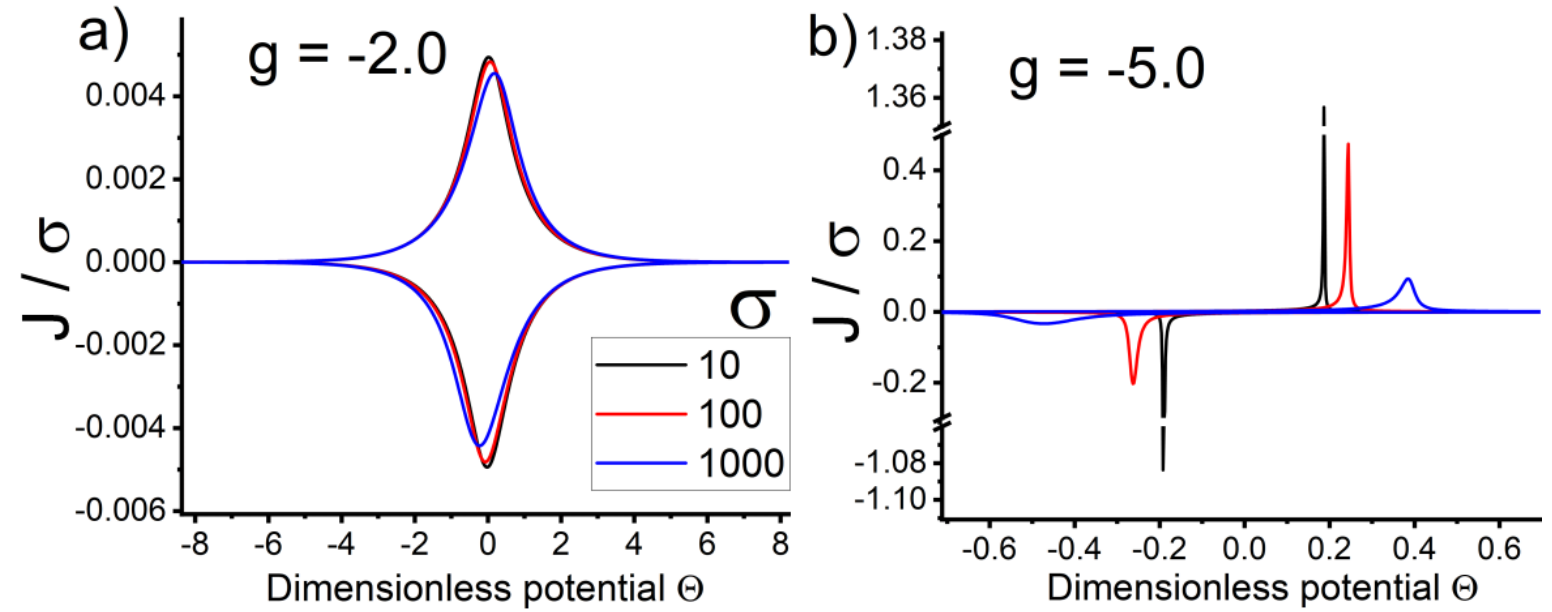

Figure 8. Simulated voltammograms as a function of dimensionless scan rate $\sigma$ for two cases of non-ideal surface interaction a) $g=-2.0$ and b) $g=-5.0$. The values of $\sigma$ and $g$ are shown in the figure. Other simulation parameters: $K_{0}^{\prime}=10^{6}, B=$ $100, \alpha=0.5$ and $g^{\ddagger}=0$.

Next, the effects of the saturation parameter $\mathrm{B}\left(\propto c_{\mathrm{A}, \text { bulk }} / \Gamma_{\text {max }}\right)$ under reversible electrode kinetics studied for $g=-2.0$ and $g=-5.0$ are shown in Figure 9 a) and b) respectively. For the electrosorption voltammetry with no phase transition, Figure 9 a), varying $B$ from 100 to 10,000 does not affect the voltammetric shape. The variation of saturation parameter $B$ can be interpreted as the change in the experimental variable $c_{\mathrm{A}, b u l k}$ since $\Gamma_{\max }$ is usually constant. The voltammogram was not affected by the increase of $B$ over two orders of magnitudes. This is because, already at the lowest saturation parameter studied ( $B=100$ ), under reversible electrode kinetics, the 'surface-bound' voltammetry as evident by the lack of diffusion tail precludes the depletion of $C_{\mathrm{A}, 0}$ throughout the voltammetric sweep. This, however contrasts with what is seen at a phase transition value of $g=$ -5.0 , shown in Figure $9 \mathrm{~b}$ ). Here, an increase in $B$ results in an increase in peak flux by a similar magnitude. The peak width, although it cannot be depicted easily, is reduced with the increase of $B$ since the area under the peaks are necessarily the same. 

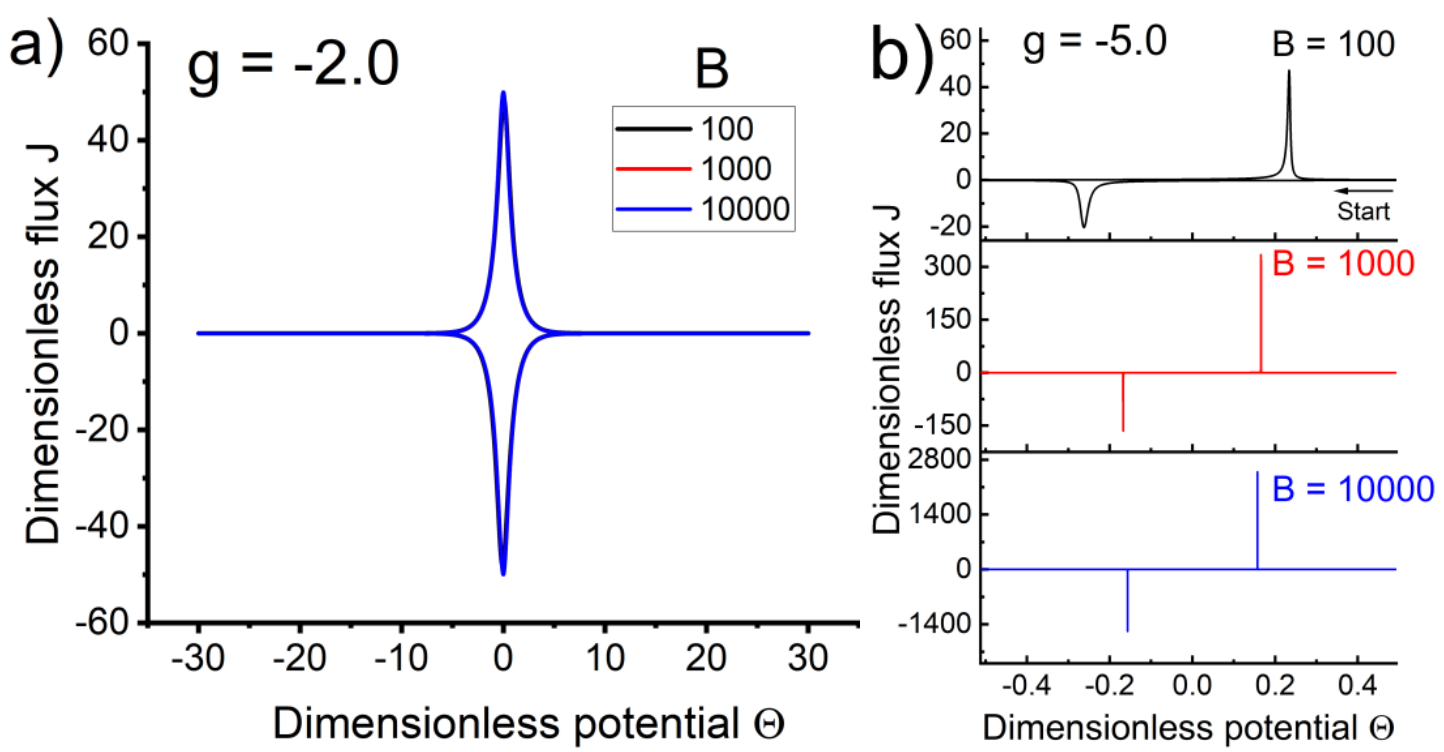

Figure 9) Simulated voltammograms as a function of $B$ for two cases of non-ideal surface interaction a) non-phase transition $g=-2.0$ and $b$ ) phase transition $g=-5.0$. The values of $g$ and $B$ are shown in the figure. Other simulation parameters $K_{0}^{\prime}=1 E 6, \sigma=100, \alpha=0.5$ and $g^{\ddagger}=0$. The three lines coincide in $a$ ). The potential range for $b$ ) was reduced to $-5.0 \leq$ $\Theta \leq+5.0$ to improve temporal resolution; only part of the $C V$ is displayed to improve visualisation.

To understand the effect of $B$ has on the voltammetry with surface interactions leading to a phase transition, the simulation data in Figure $9 \mathrm{~b}$ ) are shown in Figure 10 in a plot of $\theta_{\mathrm{B}}$ against $\frac{K C_{\mathrm{A}, 0}}{K_{\theta_{\mathrm{B}}=0.5}}$ during the forward sweep. The solid line is the corresponding analytical prediction from Frumkin adsorption isotherm. As can be seen, simulation data obtained for all values of $B$ studied in Figure 9 b) are identical and with an excellent agreement to the adsorption isotherm (as does the backward sweep, shown in SI Figure 6). How then is this possible if the peak width and peak flux in the obtained voltammogram is different? It important to note that, the $\mathrm{x}$ - and $\mathrm{y}$-axis in Figure $10, \theta_{\mathrm{B}}$ against $\frac{K C_{\mathrm{A}, 0}}{K_{\theta_{\mathrm{B}}=0.5}}$ does not reflect the timescale of the forward deposition process. The dimensionless equilibrium constant $K$ for the electro-sorption reaction is exponentially dependant on the dimensionless potential $\Theta$, and thus since the voltage is scanned also time dependant. However, the rate and magnitude of change in the interfacial $C_{\mathrm{A}, 0}$ during the voltammetry sweep is different for each value of $B$, shown in Figure 11. During the forward sweep $\left(0 \leq \frac{T}{T_{\max }}<0.5\right)$, a drop in $C_{\mathrm{A}, 0}$ are seen around $\frac{T}{T_{\max }}=0.2$ and, on the reverse sweep, a sharp increase in $C_{\mathrm{A}, 0}$ are seen around $\frac{T}{T_{\max }}=0.8$. Inlay shows a zoom-in depicting the magnitude and the timescale of concentration change during the 
forward sweep. The minimum depletion of $C_{\mathrm{A}, 0}$ for $B=100,1000$ and 10000 is from 1.0 to $0.66,0.72$ and 0.73 respectively. Moreover, from the adsorption isotherm for $g=-5.0$, the recession of the 'Sshaped' transient occurs between $x$-axis values of 1.17 and 0.86 . In the limiting case where the phase transition occurs instantaneously such that changes in $K$, with time, is assumed constant, the drop in $C_{\mathrm{A}, 0}$ during the forward sweep is has to be a minimum of $26 \%\left(\frac{1.17-0.86}{1.17}\right)$. Thus, $C_{\mathrm{A}, 0}$ does not equate to $C_{\mathrm{A}, \text { bulk }}$ at all times as the dynamic voltammetric waveforms necessary perturbs the interfacial concentration. The timescale at which phase transition occurs are reflected by the magnitude of the full width half maximum (FWHM) in the drop of $C_{\mathrm{A}, 0}$ with time, shown in the inlay of Figure 11 . As can be seen, increasing $B$ from 100 to 10000 leads to the phase transition timescale $T / T_{\max }$ from $10^{-3}$ to $10^{-}$ 7.

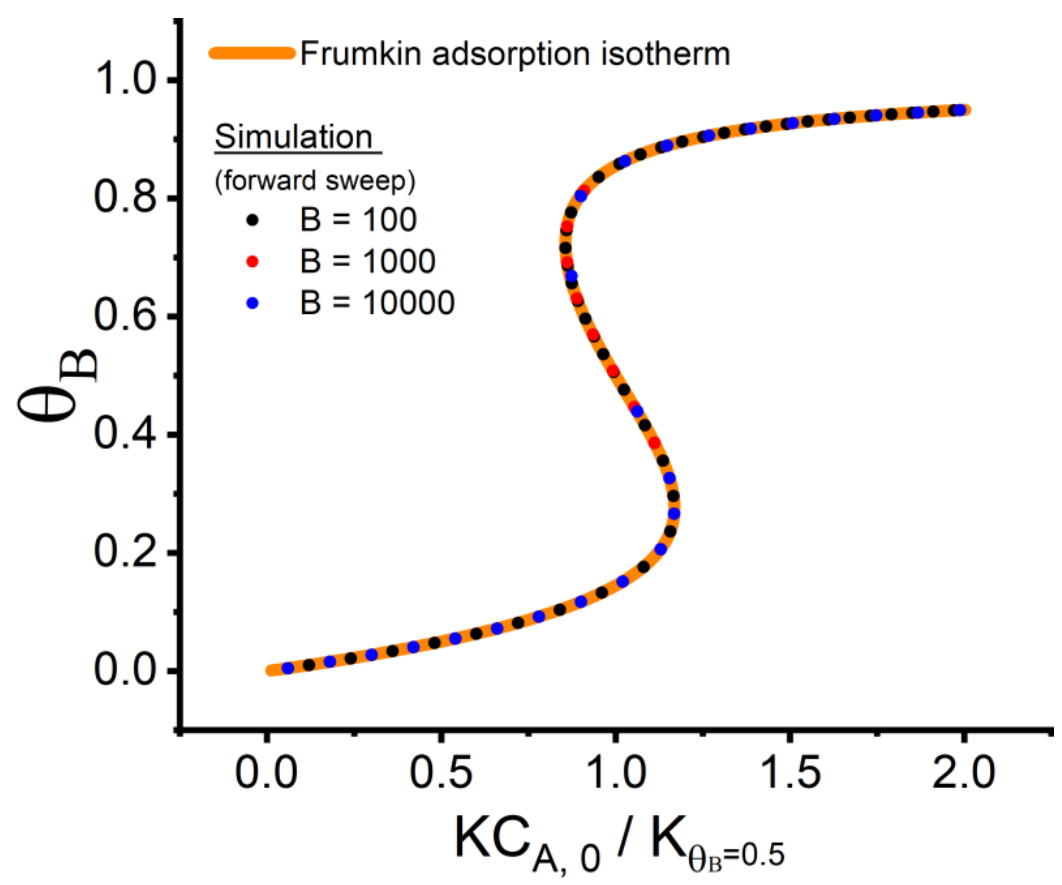

Figure 10) $A$ plot of $\theta_{\mathrm{B}}$ against $K C_{\mathrm{A}, 0} / K_{\theta_{\mathrm{B}}=0.5}$ during the forward voltammetry sweep for adsorbate-adsorbate interaction parameter $g=-5.0$. Solid line - Frumkin adsorption isotherm; circles - simulation data varying B from 100 to 10000 (CV shown in Figure $7 \mathrm{~b}$ ). The values of $B$ are shown in the figure. Other simulation parameters: $K_{0}^{\prime}=1 E 6, \sigma=100, \alpha=0.5, g=$ -5.0 and $g^{\ddagger}=0$. 


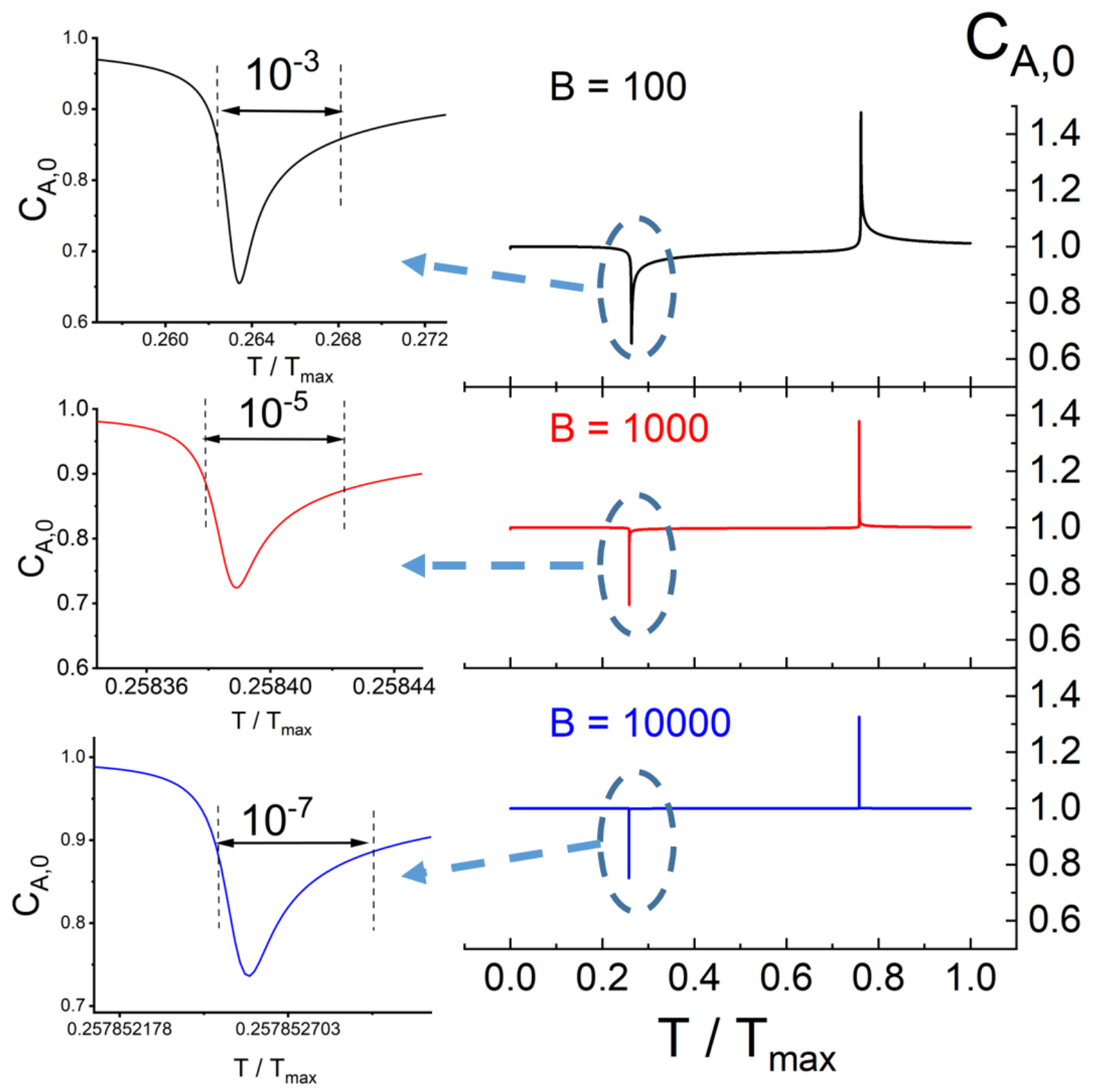

Figure 11) A plot of interfacial concentration $C_{\mathrm{A}, 0}$ for values of saturation parameter $B$ ranging from 100 to 10,000 over the time scale of voltammetry with $g$ equal to -5.0. The corresponding CV can be seen in Figure $9 \mathrm{~b}$. The values of $B$ are shown in the figure. Other simulation parameters: $K_{0}^{\prime}=1 E 6, \sigma=100, \alpha=0.5, g=-5.0$ and $g^{\ddagger}=0$. The inlay shows a zoom-in of the drop in $C_{A, 0}$ during the forward sweep demonstrating the difference in the timescale.

\section{Conclusions}

In this article, we investigated a one-electron electro-sorption mechanism where the surface interactions are assumed non-ideal. The interfacial kinetics is described by Butler-Volmer kinetics coupled with a Frumkin adsorption isotherm and with a bespoke formal potential, newly defined. 
Herein, new physicochemical insights to phase transition behaviour under voltammetry conditions are provided from a simulation perspective. We deployed the novel use of a dimensionless equilibrium constant allowing the interfacial conditions during the voltammetry simulation to be compared with the Frumkin adsorption isotherm thus providing a new ground to understand the feasibility of voltammetric induced phase transitions. This directly leads to the observation that, fast and reversible electrode kinetics are essential in capturing the voltammetric induced phase transition leading to the sharp and narrow peaks, which otherwise, would lead to a 'disconnection' from the Frumkin adsorption isotherm.

Accounting for the non-equilibrium in mass transport when in analysing the voltammetry involving non-surface interactions is essential, particularly in the cases leading to phase transition. It is precisely this non-equilibrium in mass transport that causes the interfacial reaction to transit into the 'forbidden zone' of the Frumkin adsorption isotherm in voltammetry. Even if in the case of significant excess in bulk concentration the interfacial changes in concentration is non-negligible during a voltammetric induced phase transition; we have shown that under reversible electrode kinetics, at least a $26 \%$ drop in the interfacial concentration $C_{\mathrm{A}, 0}$ is seen for $g=-5.0$. Thus, analysing voltammetric peak widths by assuming a diffusionless system, $C_{\mathrm{A}, 0}=C_{\mathrm{A}, \text { bulk }}$ at all times, may lead to an underestimate of the value of $g$.

\section{Supporting Information}

The followings are provided in Supporting Information:

- Derivation for the standard electro-sorption rate constant $\boldsymbol{k}_{\mathbf{0}}^{\prime}$.

- Simulation results for limiting Case One and Case Two.

- Effects of $g^{\ddagger}$ under fast and reversible electrode kinetics

- Effects of B on the Backward Voltammetry Sweep 


\section{Conflicts of interest}

There are no conflicts to declare.

\section{Acknowledgements}

The research leading to these results has received partial funding via an EPSRC Industrial CASE award (EP/N509711/1).

\section{Reference}

1. Damaskin, B. Adsorption of Organic Compounds on Electrodes; Springer US, 2012.

2. Baikerikar, K.; Sathyanarayana, S. Adsorption of Camphor, Camphene, Pinene, Naphthalene and Nonylic Acid at the Mercury-Solution Interface. J. Electroanal. Chem. Interfacial Electrochem. 1970, 24, 333-344.

3. De Levie, R. The Dynamic Double Layer: Two-Dimensional Condensation at the Mercury-Water Interface. Chem. Rev. 1988, 88, 599-609.

4. Stradins, J. Alexander N. Frumkin and the Electrochemistry of the 20th Century. Electrochimica Acta 1997, 42, 731-736.

5. Frumkin, A.; Gorodetzkaya, A. Electrocapillary Phenomena and Layer Formation on the Surface of Liquid Gallium. Z. Phys. Chem. (Leipzig) 1928, 136, 215.

6. Frumkin, A. Surface Tension Curves of Higher Fatty Acids and the Equation of Condition of the Surface Layer. Z. phys. Chem. 1925, 116, 466-484.

7. Damaskin, B. B.; Petrii, O. A.; Batrakov, V. V. Adsorption of Organic Compounds on Electrodes; Plenum Press, 1971.

8. Foo, K. Y.; Hameed, B. H. Insights into the Modeling of Adsorption Isotherm Systems. Chem. Eng. J. 2010, 156, 2-10.

9. Limousin, G.; Gaudet, J.-P.; Charlet, L.; Szenknect, S.; Barthes, V.; Krimissa, M. Sorption Isotherms: A Review on Physical Bases, Modeling and Measurement. Appl. Geochem. 2007, 22, 249275.

10. Nikitas, P. A Critique of the Adsorption Isotherms Used in Electrochemical Processes Involving the Adsorption of Organic Compounds. J. Chem. Soc., Faraday Trans. 1 1985, 81, 1767-1787.

11. Laviron, E.; Roullier, L. General Expression of the Linear Potential Sweep Voltammogram for a Surface Redox Reaction with Interactions between the Adsorbed Molecules: Applications to Modified Electrodes. J. Electroanal. Chem. Interfacial Electrochem. 1980, 115, 65-74.

12. Laviron, E. Ac Polarography and Faradaic Impedance of Strongly Adsorbed Electroactive Species: Part li. Theoretical Study of a Quasi-Reversible Reaction in the Case of a Frumkin Isotherm. J. Electroanal. Chem. Interfacial Electrochem. 1979, 105, 25-34.

13. Laviron, E. The Use of Linear Potential Sweep Voltammetry and of Ac Voltammetry for the Study of the Surface Electrochemical Reaction of Strongly Adsorbed Systems and of Redox Modified Electrodes. J. Electroanal. Chem. Interfacial Electrochem. 1979, 100, 263-270.

14. Ivanov, V. D. Adsorption Voltammetric Peak: Approximate Calculation Algorithm Taking into Account Lateral Interactions. J. Solid State Electrochem. 2019, 23, 1371-1377.

15. Herrero, E.; Buller, L. J.; Abruña, H. D. Underpotential Deposition at Single Crystal Surfaces of $\mathrm{Au}, \mathrm{Pt}, \mathrm{Ag}$ and Other Materials. Chem. Rev. 2001, 101, 1897-1930. 
16. Chen, R.; Najarian, A. M.; Kurapati, N.; Balla, R. J.; Oleinick, A.; Svir, I.; Amatore, C.; McCreery, R. L.; Amemiya, S. Self-Inhibitory Electron Transfer of the Co (Iii)/Co (li)-Complex Redox Couple at Pristine Carbon Electrode. Anal. Chem. 2018, 90, 11115-11123.

17. Kurapati, N.; Pathirathna, P.; Chen, R.; Amemiya, S. Voltammetric Measurement of Adsorption Isotherm for Ferrocene Derivatives on Highly Oriented Pyrolytic Graphite. Anal. Chem. 2018, 90, $13632-13639$.

18. Will, F. G. Hydrogen Adsorption on Platinum Single Crystal Electrodes: I. Isotherms and Heats of Adsorption. J. Electrochem. Soc. 1965, 112, 451.

19. Angerstein-Kozlowska, H.; Conway, B. Evaluation of Rate Constants and Reversibility Parameters for Surface Reactions by the Potential-Sweep Method. J. Electroanal. Chem. Interfacial Electrochem. 1979, 95, 1-28.

20. Gómez, R.; Orts, J. M.; Álvarez-Ruiz, B.; Feliu, J. M. Effect of Temperature on Hydrogen Adsorption on Pt (111), Pt (110), and Pt (100) Electrodes in 0.1 M Hclo4. J. Phys. Chem. B 2004, 108, 228-238.

21. White, J.; Abruna, H. Coadsorption of Copper with Anions on Platinum (111): The Role of Surface Redox Chemistry in Determining the Stability of a Metal Monolayer. J. Phys. Chem. 1990, 94, 894-900.

22. Scortichini, C.; Woodward, F.; Reilley, C. N. Surface Characterization of Pt Electrodes Using Underpotential Deposition of $\mathrm{H}$ and $\mathrm{Cu}$ : Part lii. Surface Improvement of the Flame-Annealed Pt (100) and Pt (111) Electrodes Via Potential Cycling. J. Electroanal. Chem. Interfacial Electrochem. 1982, 139, 265-274.

23. Scortichini, C. L.; Reilley, C. N. Surface Characterization of Pt Electrodes Using Underpotential Deposition of H and Cu: Part li. Pt (110) and Pt (111). J. Electroanal. Chem. Interfacial Electrochem. 1982, 139, 247-264.

24. Markovic, N.; Ross, P. Effect of Anions on the Underpotential Deposition of Copper on Platinum (111) and Platinum (100) Surfaces. Langmuir 1993, 9, 580-590.

25. Zelenay, P.; Gamboa-Aldeco, M.; Horanyi, G.; Wieckowski, A. Adsorption of Anions on Ultrathin Metal Deposits on Single-Crystal Electrodes: Part 3. Voltammetric and Radiochemical Study of Bisulfate Adsorption on Pt (111) and Pt (Poly) Electrodes Containing Silver Adatoms. J. Electroanal. Chem. 1993, 357, 307-326.

26. El Omar, F.; Durand, R.; Faure, R. Underpotential Deposition of Ag on Pt (111). Pt (100) and Pt (110) Electrodes. J. Electroanal. Chem. Interfacial Electrochem. 1984, 160, 385-392.

27. Taylor, D.; Abruna, H. The Effects of Organic Adsorbates on the Underpotential Deposition of Silver on Pt (111) Electrodes. J. Electrochem. Soc. 1993, 140, 3402.

28. Cappadonia, M.; Linke, U.; Robinson, K.; Schmidberger, J.; Stimming, U. Anion Effects on the Cyclic Voltammetry of Copper Underpotential Deposition on Au (100). J. Electroanal. Chem. 1996, 405, 227-232.

29. Hölzle, M.; Apsel, C.; Will, T.; Kolb, D. Copper Deposition onto Au (111) in the Presence of Thiourea. J. Electrochem. Soc. 1995, 142, 3741-3749.

30. Schultze, J.; Dickertmann, D. Potentiodynamic Desorption Spectra of Metallic Monolayers of $\mathrm{Cu}, \mathrm{Bi}, \mathrm{Pb}, \mathrm{Tl}$, and Sb Adsorbed at (111),(100), and (110) Planes of Gold Electrodes. Surf. Sci. 1976, 54, 489-505.

31. Chen, C. H.; Vesecky, S. M.; Gewirth, A. A. In Situ Atomic Force Microscopy of Underpotential Deposition of Silver on Gold (111). J. Am. Chem. Soc. 1992, 114, 451-458.

32. Chen, C.-h.; Gewirth, A. A. Afm Study of the Structure of Underpotentially Deposited Ag and $\mathrm{Hg}$ on $\mathrm{Au}$ (111). Ultramicroscopy 1992, 42, 437-444.

33. Mrozek, P.; Sung, Y.-e.; Han, M.; Gamboa-Aldeco, M.; Wieckowski, A.; Chen, C.-h.; Gewirth, A. A. Coadsorption of Sulfate Anions and Silver Adatoms on the Au (111) Single Crystal Electrode. Ex Situ and in Situ Comparison. Electrochim. Acta 1995, 40, 17-28.

34. Hamelin, A. Lead Adsorption on Gold Single Crystal Stepped Surfaces. J. Electroanal. Chem. Interfacial Electrochem. 1979, 101, 285-290. 
35. Hamelin, A.; Katayama, A. Lead Underpotential Deposition on Gold Single-Crystal Surfaces: The (100) Face and Its Vicinal Faces. J. Electroanal. Chem. Interfacial Electrochem. 1981, 117, 221-232. 36. Hamelin, A. Underpotential Deposition of Lead on Single Crystal Faces of Gold: Part I. The Influence of Crystallographic Orientation of the Substrate. J. Electroanal. Chem. Interfacial Electrochem. 1984, 165, 167-180.

37. Herrero, E.; Abruna, H. Underpotential Deposition of Mercury on Au (111): Electrochemical Studies and Comparison with Structural Investigations. Langmuir 1997, 13, 4446-4453.

38. Herrero, E.; Abruna, H. Anion Effects on the Kinetics of Mercury Underpotential Deposition on $\mathrm{Au}$ (111) Electrodes. J. Phys. Chem. B 1998, 102, 444-451.

39. Inukai, J.; Sugita, S.; Itaya, K. Underpotential Deposition of Mercury on Au (111) Investigated by in Situ Scanning Tunnelling Microscopy. J. Electroanal. Chem. 1996, 403, 159-168.

40. Bewick, A.; Thomas, B. Optical and Electrochemical Studies of the Underpotential Deposition of Metals Part I. Thallium Deposition on Single Crystal Silver Electrodes. J. Electroanal. Chem. Interfacial Electrochem. 1975, 65, 911-931.

41. Schultze, J.; Brenske, K.-R. Adsorption of Bismuth on Silver Single Crystal Planes and the Catalysis by Chloride Ions. J. Electroanal. Chem. Interfacial Electrochem. 1982, 137, 331-339.

42. Bewick, A.; Thomas, B. Optical and Electrochemical Studies of the under-Potential Deposition of Metals: Part lii. Lead Deposition on Silver Single Crystals. J. Electroanal. Chem. Interfacial Electrochem. 1977, 84, 127-140.

43. Bewick, A.; Thomas, B. Optical and Electrochemical Studies of the Underpotential Deposition of Metals: Part li. Phase Transitions and Two-Dimensional Nucleation. J. Electroanal. Chem. Interfacial Electrochem. 1977, 85, 329-337.

44. Schmidt, U.; Vinzelberg, S.; Staikov, G. Pb Upd on Ag (100) and Au (100) -2d Phase Formation Studied by in Situ Stm. Surf. Sci. 1996, 348, 261-279.

45. Peter, L.; Reid, J.; Scharifker, B. Electrochemical Adsorption and Phase Formation on Mercury in Sulphide Ion Solutions. J. Electroanal. Chem. Interfacial Electrochem. 1981, 119, 73-91.

46. Benucci, C.; Scharifker, B. Effect of Temperature on the Formation of Two Dimensional Sulphide Phases on Mercury. J. Electroanal. Chem. Interfacial Electrochem. 1985, 190, 199-212.

47. Philipp, R.; Retter, U. On the Anodic Film Formation of Hgs. Thin Solid Films 1992, 207, 42-50.

48. Sadkowski, A. On the Problem of Phase Transitions in Electrochemical Adsorption Reactions. J. Electroanal. Chem. Interfacial Electrochem. 1986, 208, 69-76.

49. Angerstein-Kozlowska, H.; Klinger, J.; Conway, B. Computer Simulation of the Kinetic Behaviour of Surface Reactions Driven by a Linear Potential Sweep: Part I. Model 1-Electron Reaction with a Single Adsorbed Species. J. Electroanal. Chem. Interfacial Electrochem. 1977, 75, 45-60.

50. Szulborska, A.; Baranski, A. Numerical Simulation of Kinetically Controlled Electrosorption Processes under Cyclic Voltammetric Conditions. J. Electroanal. Chem. 1994, 377, 23-31.

51. Calvente, J.-J.; Kováčová, Z.; Andreu, R.; Fawcett, W. R. Numerical Simulation of Desorption Transients at Electrodes on the Basis of Non-Linear Adsorption Isotherms. J. Chem. Soc., Faraday Trans. 1996, 92, 3701-3708.

52. Langmuir, I. The Adsorption of Gases on Plane Surfaces of Glass, Mica and Platinum. J. Am. Chem. Soc. 1918, 40, 1361-1403.

53. Kaganovich, R. E Gerovich, Vm:(Adsorption of Aliphatic Amines and Alcohols at the SolutionAir and Solution-Mercury Interfaces). Elektrokhimiya 1966, 2, 977-81.

54. Kaganovich, R.; Gerovich, V.; Osotova, T. G. Adsorption of Fatty Acids at the Solution Interface with Air and Mercury. Doklady Akademii Nauk 1964, 155, 893-896.

55. Frumkin, A.; Williams, J. W. The Relation between the Electric Moment and the Potential Difference at an Interface. Proc. Natl. Acad. Sci. U. S. A. 1929, 15, 400.

56. Butler, J. A. V. The Equilibrium of Heterogeneous Systems Including Electrolytes. Part lii. The Effect of an Electric Field on the Adsorption of Organic Molecules, and the Interpretation of ElectroCapillary Curves. Proc. R. Soc. London, Ser. A 1929, 122, 399-416.

57. Ockrent, C.; Butler, J. Studies in Electrocapillarity. Ii. J. Phys. Chem. 2002, 34, 2297-2306. 
58. Gerovich, M.; Olman, O. Elektricheskie Svoistva Plenok Kondensirovannykh Aromaticheskikh Uglevodorodov Na Vodnykh Rastvorakh Neorganicheskikh Solei. Zhurnal Fizicheskoi Khimii 1954, 28, 19-25.

59. Gerovich, M. Adsorptsya Kontsentrirovanikh Aromaticheskikh Uglevodorodow Na Gra-Nitse Rtut-Rostvor. Dokl. Akad. Nauk SSSR 1954, 96, 543.

60. Gerovich, M. Osobennosti Elektrokapillyarnogo Povedeniya Aromaticheskikh Soedinenii. Dokl. Akad. Nauk SSSR 1955, 105, 1278-1281.

61. Frumkin, A. Electrocapillary Curve of Higher Aliphatic Acids and the State Equation of the Surface Layer. Z. Phys. Chem. 1925, 116, 466-470.

62. Kirowa-Eisner, E.; Bonfil, Y.; Tzur, D.; Gileadi, E. Thermodynamics and Kinetics of Upd of Lead on Polycrystalline Silver and Gold. Journal of Electroanalytical Chemistry 2003, 552, 171-183.

63. Conway, B. E.; Gileadi, E. Kinetic Theory of Pseudo-Capacitance and Electrode Reactions at Appreciable Surface Coverage. Transactions of the Faraday Society 1962, 58, 2493-2509.

64. Hush, N. Electron Transfer in Retrospect and Prospect: 1: Adiabatic Electrode Processes. J. Electroanal. Chem. 1999, 460, 5-29.

65. Henstridge, M. C.; Laborda, E.; Compton, R. G. Asymmetric Marcus-Hush Model of Electron Transfer Kinetics: Application to the Voltammetry of Surface-Bound Redox Systems. J. Electroanal. Chem. 2012, 674, 90-96.

66. Marcus, R. A.; Sutin, N. Electron Transfers in Chemistry and Biology. Biochim. Biophys. Acta, Rev. Bioenerg. 1985, 811, 265-322.

67. Butler, J. A. V. Studies in Heterogeneous Equilibria. Part li. - the Kinetic Interpretation of the Nernst Theory of Electromotive Force. Trans. Faraday Soc. 1924, 19, 729-733.

68. Butler, J. A. V. Studies in Heterogeneous Equilibria. Part lii. A Kinetic Theory of Reversible Oxidation Potentials at Inert Electrodes. Trans. Faraday Soc. 1924, 19, 734-739.

69. Erdey-Grúz, T.; Volmer, M. Zur Theorie Der Wasserstoff Überspannung. Z. Phys. Chem. 1930, 150, 203-213.

70. Anson, F. C. The Evaluation of Surface Activities from Adsorption Isotherms and Their Utilization in Kinetic Analyses of Electrode Reactions. J. Electroanal. Chem. Interfacial Electrochem. 1973, 47, 279-285.

71. McNaught, A. D.; Wilkinson, A. Iupac. Compendium of Chemical Terminology, 2nd Ed. (the "Gold Book"); Blackwell Scientific Publications: Oxford, 1997.

72. Smith, G. D. Numerical Solution of Partial Differential Equations: Finite Difference Methods; Oxford University Press, 1985.

73. Morton, K. W.; Mayers, D. F. Numerical Solution of Partial Differential Equations. J. Fluid Mech. 1998, 363, 349-349.

74. Compton, R. G.; Eduardo, L.; Katelhon, E. Understanding Voltammetry: Simulation of Electrode Processes, Second ed.; World Scientific, 2020.

75. Nernst, W. Theorie Der Reaktionsgeschwindigkeit in Heterogenen Systemen. Z. Phys. Chem. $1904,47,52-55$.

76. Svir, I.; Oleinick, A.; Compton, R. Solution of Ring Electrode Problems in Spherical Coordinates: An Application to near-Steady-State Linear Sweep Voltammetry. Russ. J. Electrochem. 2003, 39, 160164.

77. Kätelhön, E.; Compton, R. G. Testing and Validating Electroanalytical Simulations. Analyst 2015, 140, 2592-2598.

78. Randles, J. E. A Cathode Ray Polarograph. Part li.-the Current-Voltage Curves. Trans. Faraday Soc. 1948, 44, 327-338.

79. Ševčik, A. Oscillographic Polarography with Periodical Triangular Voltage. Collect. Czech. Chem. Commun. 1948, 13, 349-377.

80. Compton, R. G.; Banks, C. E. Understanding Voltammetry, third ed.; World Scientific, 2018.

81. Matsuda, H.; Ayabe, Y. Electrolytic Preparation of Titanium from Fused Salts. Electrochemistry 1955, 59, 494. 


\section{TOC Graphic}

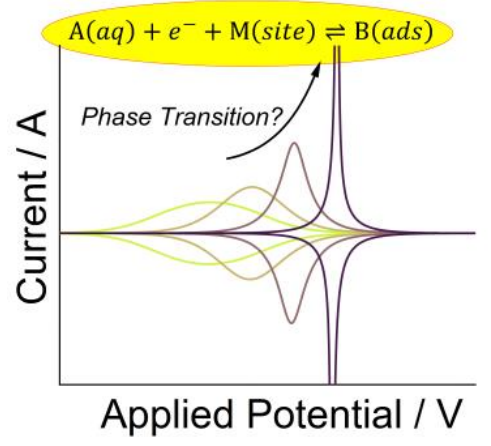

